\title{
ON POINCARÉ SERIES OF HALF-INTEGRAL WEIGHT
}

\author{
SONJA ŽUnAR \\ University of Zagreb, Croatia
}

\begin{abstract}
We use Poincaré series of $K$-finite matrix coefficients of genuine integrable representations of the metaplectic cover of $\mathrm{SL}_{2}(\mathbb{R})$ to construct a spanning set for the space of cusp forms $S_{m}(\Gamma, \chi)$, where $\Gamma$ is a discrete subgroup of finite covolume in the metaplectic cover of $\mathrm{SL}_{2}(\mathbb{R}), \chi$ is a character of $\Gamma$ of finite order, and $m \in \frac{5}{2}+\mathbb{Z}_{\geq 0}$. We give a result on the non-vanishing of the constructed cusp forms and compute their Petersson inner product with any $f \in S_{m}(\Gamma, \chi)$. Using this last result, we construct a Poincaré series $\Delta_{\Gamma, k, m, \xi, \chi} \in S_{m}(\Gamma, \chi)$ that corresponds, in the sense of the Riesz representation theorem, to the linear functional $f \mapsto f^{(k)}(\xi)$ on $S_{m}(\Gamma, \chi)$, where $\xi \in \mathbb{C}_{\Im(z)>0}$ and $k \in \mathbb{Z}_{\geq 0}$. Under some additional conditions on $\Gamma$ and $\chi$, we provide the Fourier expansion of cusp forms $\Delta_{\Gamma, k, m, \xi, \chi}$ and their expansion in a series of classical Poincaré series.
\end{abstract}

\section{INTRODUCTION}

In this paper, we adapt representation-theoretic techniques developed for the group $\mathrm{SL}_{2}(\mathbb{R})$ in [5] and [9] to the case of the metaplectic cover of $\mathrm{SL}_{2}(\mathbb{R})$. Using this, we prove a few results on cusp forms of half-integral weight.

To give an overview of our results, we introduce the basic notation. The metaplectic cover of $\mathrm{SL}_{2}(\mathbb{R})$ can be realized as the group

$$
\begin{aligned}
\mathrm{SL}_{2}(\mathbb{R})^{\sim}:= & \left\{\sigma=\left(g_{\sigma}=\left(\begin{array}{ll}
a_{\sigma} & b_{\sigma} \\
c_{\sigma} & d_{\sigma}
\end{array}\right), \eta_{\sigma}\right) \in \mathrm{SL}_{2}(\mathbb{R}) \times \operatorname{Hol}(\mathcal{H}):\right. \\
& \left.\eta_{\sigma}^{2}(z)=c_{\sigma} z+d_{\sigma} \text { for all } z \in \mathcal{H}\right\},
\end{aligned}
$$

2010 Mathematics Subject Classification. 11F12, 11F37.

Key words and phrases. Cusp forms of half-integral weight, Poincaré series, metaplectic cover of $\mathrm{SL}_{2}(\mathbb{R})$

The author acknowledges Croatian Science Foundation Grant No. 9364. 
where $\operatorname{Hol}(\mathcal{H})$ is the space of all holomorphic functions defined on the upper half-plane $\mathcal{H}$. The multiplication law and smooth structure of $\mathrm{SL}_{2}(\mathbb{R}) \sim$ are defined in Section 2. The group $\mathrm{SL}_{2}(\mathbb{R})^{\sim}$ acts on $\mathbb{C} \cup\{\infty\}$ by $\sigma . z:=\frac{a_{\sigma} z+b_{\sigma}}{c_{\sigma} z+d_{\sigma}}$. Moreover, let us denote by $\mathbb{C}^{\mathcal{H}}$ the set of all functions $\mathcal{H} \rightarrow \mathbb{C}$. For every $m \in$ $\frac{1}{2}+\mathbb{Z}_{\geq 0}$, we have the following right action of $\mathrm{SL}_{2}(\mathbb{R})^{\sim}$ on $\mathbb{C}^{\mathcal{H}}:\left(\left.f\right|_{m} \sigma\right)(z):=$ $f(\sigma . z) \eta_{\sigma}(z)^{-2 m}$. Let $P: \mathrm{SL}_{2}(\mathbb{R})^{\sim} \rightarrow \mathrm{SL}_{2}(\mathbb{R})$ be the projection onto the first coordinate.

Next, let $\Gamma$ be a discrete subgroup of finite covolume in $\mathrm{SL}_{2}(\mathbb{R})^{\sim}, \chi$ : $\Gamma \rightarrow \mathbb{C}^{\times}$a character of finite order, and $m \in \frac{3}{2}+\mathbb{Z}_{\geq 0}$. The space $S_{m}(\Gamma, \chi)$ of cusp forms of weight $m$ for $\Gamma$ with character $\chi$ by definition consists of all $f \in \operatorname{Hol}(\mathcal{H})$ that satisfy $\left.f\right|_{m} \gamma=\chi(\gamma) f$ for all $\gamma \in \Gamma$ and vanish at all cusps of $P(\Gamma)$. The space $S_{m}(\Gamma, \chi)$ is a finite-dimensional Hilbert space under the Petersson inner product

$$
\left\langle f_{1}, f_{2}\right\rangle_{\Gamma}:=\left|\Gamma \cap Z\left(\mathrm{SL}_{2}(\mathbb{R})^{\sim}\right)\right|^{-1} \int_{\Gamma \backslash \mathcal{H}} f_{1}(z) \overline{f_{2}(z)} \Im(z)^{m} d v(z),
$$

where $d v(x+i y):=\frac{d x d y}{y^{2}}$. We write $S_{m}(\Gamma):=S_{m}(\Gamma, 1)$. Let us denote by $\Phi$ the classical lift $S_{m}(\Gamma) \rightarrow \mathbb{C}^{\mathrm{SL}_{2}(\mathbb{R})^{\sim}}$ that maps $f \in S_{m}(\Gamma)$ to

$$
F_{f}: \mathrm{SL}_{2}(\mathbb{R})^{\sim} \rightarrow \mathbb{C}, \quad F_{f}(\sigma):=\left(\left.f\right|_{m} \sigma\right)(i),
$$

where $i$ is the imaginary unit. The function $\Phi$ is a unitary isomorphism $S_{m}(\Gamma) \rightarrow \Phi\left(S_{m}(\Gamma)\right)=: \mathcal{A}\left(\Gamma \backslash \mathrm{SL}_{2}(\mathbb{R})^{\sim}\right)_{m} \subseteq L^{2}\left(\Gamma \backslash \mathrm{SL}_{2}(\mathbb{R})^{\sim}\right)$ (Theorem 4.3).

The starting point of this paper are results of [12], where we applied the techniques of [5] to compute certain $K$-finite matrix coefficients of genuine integrable representations of $\mathrm{SL}_{2}(\mathbb{R})^{\sim}$ and study their Poincaré series with respect to $\Gamma$. In Lemma 5.1.(5), we show that the Poincaré series $P_{\Gamma} F_{k, m}(k \in$ $\left.\mathbb{Z}_{\geq 0}, m \in \frac{5}{2}+\mathbb{Z}_{\geq 0}\right)$ discussed in $[12$, Section 6$]$ belong to $\mathcal{A}\left(\Gamma \backslash \mathrm{SL}_{2}(\mathbb{R})^{\sim}\right)_{m}$. The main result of this paper is Theorem 5.4, which we call The inner product formula. It is the formula for the Petersson inner product of $P_{\Gamma} F_{k, m}$ with any $\varphi \in \mathcal{A}\left(\Gamma \backslash \mathrm{SL}_{2}(\mathbb{R})^{\sim}\right)_{m}$. We prove it using the representation theory of $\mathrm{SL}_{2}(\mathbb{R})^{\sim}$. It is the $\mathrm{SL}_{2}(\mathbb{R})^{\sim}$-variant of $[9$, Theorem 2-11].

In the rest of the paper, we use the facts of the previous paragraph to prove a few results about $S_{m}(\Gamma, \chi)$ for $m \in \frac{5}{2}+\mathbb{Z}_{\geq 0}$. Most of these results are half-integral weight variants of results of $[5],[6],[7]$, and [9].

First, by considering the preimages of functions $P_{\Gamma} F_{k, m}$ under $\Phi$, in Theorem 6.1 we construct the following spanning set for $S_{m}(\Gamma, \chi)$ (cf. [5, Lemma $4-2])$ :

$$
\left(P_{\Gamma, \chi} f_{k, m}\right)(z):=(2 i)^{m} \sum_{\gamma \in \Gamma} \overline{\chi(\gamma)} \frac{(\gamma \cdot z-i)^{k}}{(\gamma \cdot z+i)^{m+k}} \eta_{\gamma}(z)^{-2 m}, \quad z \in \mathcal{H}, k \in \mathbb{Z}_{\geq 0},
$$

(see (2.1)). Moreover, we obtain results (Theorem 6.2 and Corollary 6.3) on the non-vanishing of cusp forms $P_{\Gamma, \chi} f_{k, m}$ in the case when $P(\Gamma) \subseteq \mathrm{SL}_{2}(\mathbb{Z})$ 
by adapting our study of the non-vanishing of functions $P_{\Gamma} F_{k, m}$ conducted in [12, Section 6].

Next, The inner product formula translates via the unitary isomorphism $\Phi^{-1}$ to Theorem 6.1.(3), which states that for every $k \in \mathbb{Z}_{\geq 0}$,

$$
\left\langle f, P_{\Gamma, \chi} f_{k, m}\right\rangle_{\Gamma}=\sum_{l=0}^{k}\left(\begin{array}{l}
k \\
l
\end{array}\right)(2 i)^{l} \frac{4 \pi}{\prod_{r=0}^{l}(m-1+r)} f^{(l)}(i), \quad f \in S_{m}(\Gamma, \chi) .
$$

It is a short way from this relation to the proof of the following fact in the case when $\xi=i$ : for every $k \in \mathbb{Z}_{\geq 0}$, the Poincaré series

$\Delta_{\Gamma, k, m, \xi, \chi}(z):=\frac{(2 i)^{m}}{4 \pi}\left(\prod_{r=0}^{k}(m-1+r)\right) \sum_{\gamma \in \Gamma} \frac{\overline{\chi(\gamma)}}{(\gamma \cdot z-\bar{\xi})^{m+k}} \eta_{\gamma}(z)^{-2 m}, z \in \mathcal{H}$

belongs to $S_{m}(\Gamma, \chi)$ and satisfies

$$
\left\langle f, \Delta_{\Gamma, k, m, \xi, \chi}\right\rangle_{\Gamma}=f^{(k)}(\xi), \quad f \in S_{m}(\Gamma, \chi) .
$$

We prove that this holds for all $\xi \in \mathcal{H}$ in Proposition 7.1 and Theorem 7.4 (cf. [9, Corollary 1-2]).

Incidentally, our proof of Theorem 7.4 proves the following integral formula (Corollary 7.5):

$$
f^{(k)}(\xi)=\frac{(-2 i)^{m}}{4 \pi}\left(\prod_{r=0}^{k}(m-1+r)\right) \int_{\mathcal{H}} \frac{f(z)}{(\bar{z}-\xi)^{m+k}} \Im(z)^{m} d v(z)
$$

for all $f \in S_{m}(\Gamma, \chi), k \in \mathbb{Z}_{\geq 0}$, and $\xi \in \mathcal{H}$. We use this formula in Corollary 7.6 to give a short proof that

$$
\sup _{\xi \in \mathcal{H}}\left|f^{(k)}(\xi) \Im(\xi)^{\frac{m}{2}+k}\right|<\infty, \quad f \in S_{m}(\Gamma, \chi), k \in \mathbb{Z}_{\geq 0}
$$

which enables us to prove, in Proposition 7.7, that

$$
\sup _{z, \xi \in \mathcal{H}} \Im(\xi)^{\frac{m}{2}+k} \Im(z)^{\frac{m}{2}}\left|\Delta_{\Gamma, k, m, \xi, \chi}(z)\right|<\infty
$$

for every $k \in \mathbb{Z}_{\geq 0}$ (cf. [7, (1-5)]).

Next, assume that $\infty$ is a cusp of $P(\Gamma)$ and that $\eta_{\gamma}^{-2 m}=\chi(\gamma)$ for all $\gamma \in \Gamma_{\infty}:=\{\gamma \in \Gamma: \gamma \cdot \infty=\infty\}$, so that we have the classical Poincaré series $\psi_{\Gamma, n, m, \chi} \in S_{m}(\Gamma, \chi), n \in \mathbb{Z}_{>0}$, defined by

$$
\psi_{\Gamma, n, m, \chi}(z):=\sum_{\gamma \in \Gamma_{\infty} \backslash \Gamma} \overline{\chi(\gamma)} e^{2 \pi i n \frac{\gamma \cdot z}{h}} \eta_{\gamma}(z)^{-2 m}, \quad z \in \mathcal{H}
$$

where $h \in \mathbb{R}_{>0}$ is such that the group $\{ \pm 1\} P\left(\Gamma_{\infty}\right)$ is generated by $\left\{ \pm\left(\begin{array}{cc}1 & h \\ & 1\end{array}\right)\right\}$. Theorem 8.2 gives the Fourier expansion of cusp forms $\Delta_{\Gamma, k, m, \xi, \chi}$ and their expansion in a series of classical Poincaré series (cf. [6, Theorem 3-5]). In Corollary 8.3, this Fourier expansion combined with 
(1.2) provides a quick proof of some bounds on the derivatives of classical Poincaré series (cf. [7, Theorem 1-2]).

Finally, in Section 9 we apply our results to the standardly defined spaces $S_{m}(N, \chi)$, where $N \in 4 \mathbb{Z}_{>0}$ and $\chi$ is an even Dirichlet character modulo $N$ (e.g., see [11]). We show that $S_{m}(N, \chi)$ coincides with $S_{m}\left(\boldsymbol{\Gamma}_{0}(N), \chi\right)$, where $\boldsymbol{\Gamma}_{0}(N)$ is an appropriate discrete subgroup of $\mathrm{SL}_{2}(\mathbb{R})^{\sim}$, and $\chi$ is identified with a suitable character of $\boldsymbol{\Gamma}_{0}(N)$. Corollary 9.1 gives a formula for the action of Hecke operators $T_{p^{2}, m, \chi}$, for prime numbers $p \nmid N$, on cusp forms $\Delta_{\Gamma_{0}(N), k, m, \xi, \chi}$ in terms of their expansion in a series of classical Poincaré series (cf. [6, Lemma 5-8]).

Let us mention that a non-representation-theoretic proof of formulae for cusp forms $\Delta_{\Gamma, k, m, \xi, \chi}$ (Proposition 7.1 and Theorem 7.4) in the case when $k=0$ can be obtained by adapting the proof of their integral weight version $[4$, Theorem 6.3.3] to the case of half-integral weight. The case when $k \in \mathbb{Z}_{>0}$ can be derived from the case when $k=0$ essentially by taking the $k$ th derivative (the details can be gleaned from the first sentence of the proof of Proposition 7.1 and from Lemma 7.3). Similarly, the integral formula (1.1) can be deduced from the half-integral weight variant of [4, Theorem 6.2 .2$]$; the integral weight variant of the formula (1.1) for $k=0$ is actually used in the proof of the above-mentioned [4, Theorem 6.3.3] (see the last equality on [4, pg. 230]). On the other hand, our results on the non-vanishing of cusp forms $P_{\Gamma, \chi} f_{k, m}$ are based on applying Muić's integral non-vanishing criterion [8, Lemma 2-1] to the corresponding Poincaré series on $\mathrm{SL}_{2}(\mathbb{R})^{\sim}$. To do that, we used the Cartan decomposition of $\mathrm{SL}_{2}(\mathbb{R})^{\sim}$, which is not easily accessible when working directly in $S_{m}(\Gamma, \chi)$.

\section{Preliminaries on the Metaplectic Group}

Let $\sqrt{\cdot}: \mathbb{C} \rightarrow \mathbb{C}$ be the branch of the complex square root with values in $\{z \in \mathbb{C}: \Re(z)>0\} \cup\{z \in \mathbb{C}: \Re(z)=0, \Im(z) \geq 0\}$. We write $i:=\sqrt{-1}$ and

$$
z^{m}:=(\sqrt{z})^{2 m}, \quad z \in \mathbb{C}^{\times}, m \in \frac{1}{2}+\mathbb{Z} .
$$

Next, we define $\mathcal{H}:=\{z \in \mathbb{C}: \Im(z)>0\}$ and denote by $\operatorname{Hol}(\mathcal{H})$ the space of all holomorphic functions $\mathcal{H} \rightarrow \mathbb{C}$.

The group $\mathrm{SL}_{2}(\mathbb{R})$ acts on $\mathbb{C} \cup\{\infty\}$ by

$$
g . z:=\frac{a z+b}{c z+d}, \quad g=\left(\begin{array}{ll}
a & b \\
c & d
\end{array}\right) \in \mathrm{SL}_{2}(\mathbb{R}), z \in \mathbb{C} \cup\{\infty\} .
$$

We have

$$
\Im(g . z)=\frac{\Im(z)}{|c z+d|^{2}}, \quad g=\left(\begin{array}{ll}
a & b \\
c & d
\end{array}\right) \in \mathrm{SL}_{2}(\mathbb{R}), \quad z \in \mathcal{H}
$$


For every $N \in \mathbb{Z}_{>0}$, we denote

$$
\begin{aligned}
\Gamma_{0}(N) & :=\left\{\left(\begin{array}{ll}
a & b \\
c & d
\end{array}\right) \in \mathrm{SL}_{2}(\mathbb{Z}): c \equiv 0 \quad(\bmod N)\right\}, \\
\Gamma_{1}(N): & =\left\{\left(\begin{array}{ll}
a & b \\
c & d
\end{array}\right) \in \mathrm{SL}_{2}(\mathbb{Z}): c \equiv 0, a \equiv d \equiv 1 \quad(\bmod N)\right\}, \\
\Gamma(N) & :=\left\{\left(\begin{array}{ll}
a & b \\
c & d
\end{array}\right) \in \mathrm{SL}_{2}(\mathbb{Z}): b \equiv c \equiv 0, a \equiv d \equiv 1 \quad(\bmod N)\right\} .
\end{aligned}
$$

The group

$$
\begin{aligned}
\mathrm{SL}_{2}(\mathbb{R})^{\sim}:= & \left\{\sigma=\left(g_{\sigma}=\left(\begin{array}{ll}
a_{\sigma} & b_{\sigma} \\
c_{\sigma} & d_{\sigma}
\end{array}\right), \eta_{\sigma}\right) \in \mathrm{SL}_{2}(\mathbb{R}) \times \operatorname{Hol}(\mathcal{H}):\right. \\
& \left.\eta_{\sigma}^{2}(z)=c_{\sigma} z+d_{\sigma} \text { for all } z \in \mathcal{H}\right\},
\end{aligned}
$$

with multiplication law

$$
\sigma_{1} \sigma_{2}:=\left(g_{\sigma_{1}} g_{\sigma_{2}}, \eta_{\sigma_{1}}\left(g_{\sigma_{2}} . z\right) \eta_{\sigma_{2}}(z)\right), \quad \sigma_{1}, \sigma_{2} \in \mathrm{SL}_{2}(\mathbb{R})^{\sim},
$$

acts on $\mathbb{C} \cup\{\infty\}$ by

$$
\sigma . z:=g_{\sigma} . z, \quad \sigma \in \mathrm{SL}_{2}(\mathbb{R})^{\sim}, z \in \mathbb{C} \cup\{\infty\},
$$

and, for every $m \in \frac{1}{2}+\mathbb{Z}_{\geq 0}$, on the right on $\mathbb{C}^{\mathcal{H}}$ by

$$
\left(\left.f\right|_{m} \sigma\right)(z):=f(\sigma . z) \eta_{\sigma}(z)^{-2 m}, \quad z \in \mathcal{H}, f \in \mathbb{C}^{\mathcal{H}}, \sigma \in \mathrm{SL}_{2}(\mathbb{R})^{\sim} .
$$

In the following, we use shorthand notation $\left(g_{\sigma}, \eta_{\sigma}(i)\right)$ for elements $\sigma=$ $\left(g_{\sigma}, \eta_{\sigma}\right)$ of $\mathrm{SL}_{2}(\mathbb{R})^{\sim}$. The group $\mathrm{SL}_{2}(\mathbb{R})^{\sim}$ is a connected Lie group with a smooth (Iwasawa) parametrization $\mathbb{R} \times \mathbb{R}_{>0} \times \mathbb{R} \rightarrow \mathrm{SL}_{2}(\mathbb{R})^{\sim}$,

$$
(x, y, t) \mapsto\left(\left(\begin{array}{cc}
1 & x \\
0 & 1
\end{array}\right), 1\right)\left(\left(\begin{array}{cc}
y^{\frac{1}{2}} & 0 \\
0 & y^{-\frac{1}{2}}
\end{array}\right), y^{-\frac{1}{4}}\right)\left(\left(\begin{array}{cc}
\cos t & -\sin t \\
\sin t & \cos t
\end{array}\right), e^{i \frac{t}{2}}\right) .
$$

The projection $P: \mathrm{SL}_{2}(\mathbb{R})^{\sim} \rightarrow \mathrm{SL}_{2}(\mathbb{R})$ onto the first coordinate is a smooth covering homomorphism of degree 2 . The center of $\mathrm{SL}_{2}(\mathbb{R})^{\sim}$ is $Z\left(\mathrm{SL}_{2}(\mathbb{R})^{\sim}\right):=$ $P^{-1}(\{ \pm 1\}) \cong(\mathbb{Z} / 4 \mathbb{Z},+)$.

We will denote the three factors on the right-hand side of (2.5), from left to right, by $n_{x}, a_{y}$, and $\kappa_{t}$.

The group $K:=\left\{\kappa_{t}: t \in \mathbb{R}\right\}$ is a maximal compact subgroup of $\mathrm{SL}_{2}(\mathbb{R})^{\sim}$. It is isomorphic to $(\mathbb{R} / 4 \pi \mathbb{Z},+)$ via $\kappa_{t} \mapsto t+4 \pi \mathbb{Z}$. Its unitary dual consists of the characters $\chi_{n}, n \in \frac{1}{2} \mathbb{Z}$, defined by

$$
\chi_{n}\left(\kappa_{t}\right):=e^{-i n t}, \quad t \in \mathbb{R} .
$$

We say that a function $F: \mathrm{SL}_{2}(\mathbb{R})^{\sim} \rightarrow \mathbb{C}$ transforms on the left (resp., on the right) by $K$ as $\chi_{n}$ if $F(\kappa \sigma)=\chi_{n}(\kappa) F(\sigma)$ (resp., $F(\sigma \kappa)=F(\sigma) \chi_{n}(\kappa)$ ) for all $\kappa \in K$ and $\sigma \in \mathrm{SL}_{2}(\mathbb{R})^{\sim}$. 
Let us define

$$
A^{+}:=\left\{h_{t}:=\left(\left(\begin{array}{cc}
e^{t} & 0 \\
0 & e^{-t}
\end{array}\right), e^{-\frac{t}{2}}\right): t \in \mathbb{R}_{\geq 0}\right\} .
$$

The Cartan decomposition of $\mathrm{SL}_{2}(\mathbb{R})^{\sim}$ is given by

$$
\mathrm{SL}_{2}(\mathbb{R})^{\sim}=K A^{+} K \text {. }
$$

Next, we recall the $\mathrm{SL}_{2}(\mathbb{R})$-invariant Radon measure $v$ on $\mathcal{H}$ defined by $d v(x+i y):=\frac{d x d y}{y^{2}}, x \in \mathbb{R}, y \in \mathbb{R}_{>0}$, and fix the following Haar measure on $\mathrm{SL}_{2}(\mathbb{R})^{\sim}$ : for $\varphi \in C_{c}\left(\mathrm{SL}_{2}(\mathbb{R})^{\sim}\right)$,

$$
\begin{aligned}
\int_{\mathrm{SL}_{2}(\mathbb{R}) \sim} \varphi d \mu_{\mathrm{SL}_{2}(\mathbb{R}) \sim} & :=\frac{1}{4 \pi} \int_{0}^{4 \pi} \int_{\mathcal{H}} \varphi\left(n_{x} a_{y} \kappa_{t}\right) d v(x+i y) d t \\
& =\frac{1}{4 \pi} \int_{0}^{4 \pi} \int_{0}^{\infty} \int_{0}^{4 \pi} \varphi\left(\kappa_{\theta_{1}} h_{t} \kappa_{\theta_{2}}\right) \sinh (2 t) d \theta_{1} d t d \theta_{2} .
\end{aligned}
$$

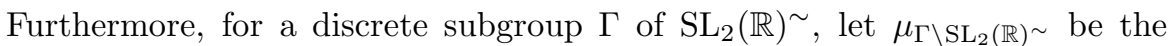
unique Radon measure on $\Gamma \backslash \mathrm{SL}_{2}(\mathbb{R})^{\sim}$ such that, for all $\varphi \in C_{c}\left(\mathrm{SL}_{2}(\mathbb{R})^{\sim}\right)$,

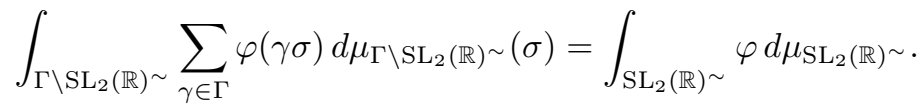

Equivalently, for all $\varphi \in C_{c}\left(\Gamma \backslash \mathrm{SL}_{2}(\mathbb{R})^{\sim}\right)$,

$$
\int_{\Gamma \backslash \mathrm{SL}_{2}(\mathbb{R}) \sim} \varphi d \mu_{\Gamma \backslash \mathrm{SL}_{2}(\mathbb{R}) \sim}=\frac{1}{4 \pi \varepsilon_{\Gamma}} \int_{0}^{4 \pi} \int_{\Gamma \backslash \mathcal{H}} \varphi\left(n_{x} a_{y} \kappa_{t}\right) d v(x+i y) d t
$$

where $\varepsilon_{\Gamma}:=\left|\Gamma \cap Z\left(\mathrm{SL}_{2}(\mathbb{R})^{\sim}\right)\right|$. For every $p \in \mathbb{R}_{\geq 1}$, we define the spaces $L^{p}\left(\mathrm{SL}_{2}(\mathbb{R})^{\sim}\right)$ and $L^{p}\left(\Gamma \backslash \mathrm{SL}_{2}(\mathbb{R})^{\sim}\right)$ using $\mu_{\mathrm{SL}_{2}(\mathbb{R}) \sim}$ and $\mu_{\Gamma \backslash \mathrm{SL}_{2}(\mathbb{R}) \sim \text {, respec- }}$ tively.

We identify the Lie algebra $\mathfrak{g}:=\operatorname{Lie}\left(\mathrm{SL}_{2}(\mathbb{R})^{\sim}\right)$ with Lie $\left(\mathrm{SL}_{2}(\mathbb{R})\right) \equiv \mathfrak{s l}_{2}(\mathbb{R})$ via the differential of $P$ at 1 and extend this identification to that of the universal enveloping algebras of their complexifications: $U\left(\mathfrak{g}_{\mathbb{C}}\right) \equiv U\left(\mathfrak{s l}_{2}(\mathbb{C})\right)$. Now,

$$
k^{\circ}:=\left(\begin{array}{cc}
0 & -i \\
i & 0
\end{array}\right), \quad n^{+}:=\frac{1}{2}\left(\begin{array}{cc}
1 & i \\
i & -1
\end{array}\right), \quad n^{-}:=\frac{1}{2}\left(\begin{array}{cc}
1 & -i \\
-i & -1
\end{array}\right)
$$

form a standard basis of $\mathfrak{g}_{\mathbb{C}}$ (we have $\left[k^{\circ}, n^{+}\right]=2 n^{+},\left[k^{\circ}, n^{-}\right]=-2 n^{-}$, and $\left.\left[n^{+}, n^{-}\right]=k^{\circ}\right)$, and

$$
\mathcal{C}:=\frac{1}{2}\left(k^{\circ}\right)^{2}+n^{+} n^{-}+n^{-} n^{+}
$$

generates the center of $U\left(\mathfrak{g}_{\mathbb{C}}\right)$. We will need the formulae [12, (2.13)-(2.14)] giving the action of $\mathcal{C}$ and $n^{+}$as left-invariant differential operators on 
$C^{\infty}\left(\mathrm{SL}_{2}(\mathbb{R})^{\sim}\right)$ in Iwasawa coordinates:

$$
\begin{aligned}
\mathcal{C} & =2 y^{2}\left(\frac{\partial^{2}}{\partial x^{2}}+\frac{\partial^{2}}{\partial y^{2}}\right)+2 y \frac{\partial^{2}}{\partial x \partial t}, \\
n^{+} & =i y e^{-2 i t}\left(\frac{\partial}{\partial x}-i \frac{\partial}{\partial y}\right)+\frac{i}{2} e^{-2 i t} \frac{\partial}{\partial t} .
\end{aligned}
$$

Clearly, $n^{-}$acts as the complex conjugate of $n^{+}$:

$$
n^{-}=-i y e^{2 i t}\left(\frac{\partial}{\partial x}+i \frac{\partial}{\partial y}\right)-\frac{i}{2} e^{2 i t} \frac{\partial}{\partial t} .
$$

\section{Preliminaries on CUSP FORMS OF HALF-INTEGRAL WEIGHT}

Let $m \in \frac{3}{2}+\mathbb{Z}_{\geq 0}$. Let $\Gamma$ be a discrete subgroup of finite covolume in $\mathrm{SL}_{2}(\mathbb{R})^{\sim}$. We denote by $S_{m}(\Gamma)$ the space of cusp forms for $\Gamma$ of weight $m$, i.e., the space of all $f \in \operatorname{Hol}(\mathcal{H})$ that satisfy

$$
\left.f\right|_{m} \gamma=f, \quad \gamma \in \Gamma
$$

and vanish at every cusp of $P(\Gamma)$. Let us explain the last condition. For a cusp $x$ of $P(\Gamma)$, let $\sigma \in \mathrm{SL}_{2}(\mathbb{R})^{\sim}$ such that $\sigma \cdot \infty=x$. Then, it follows from $[4$, Theorem 1.5.4.(2)] that

$$
Z\left(\mathrm{SL}_{2}(\mathbb{R})^{\sim}\right) \sigma^{-1} \Gamma_{x} \sigma=Z\left(\mathrm{SL}_{2}(\mathbb{R})^{\sim}\right)\left\langle n_{h}\right\rangle
$$

for some $h \in \mathbb{R}_{>0}$, hence $\left.f\right|_{m} \sigma$ has a Fourier expansion of the form

$$
\left(\left.f\right|_{m} \sigma\right)(z)=\sum_{n \in \mathbb{Z}} a_{n} e^{\pi i n \frac{z}{2 h}}, \quad z \in \mathcal{H} .
$$

We say that $f$ vanishes at $x$ if $a_{n}=0$ for all $n \in \mathbb{Z}_{\leq 0}$.

Next, we recall the half-integral weight variant of [4, Theorems 2.1 .5 and 6.3.1].

Lemma 3.1. Let $f \in \operatorname{Hol}(\mathcal{H})$ such that (3.1) holds. Then, the following claims are equivalent:

(1) $f \in S_{m}(\Gamma)$.

(2) $\sup _{z \in \mathcal{H}}\left|f(z) \Im(z)^{\frac{m}{2}}\right|<\infty$.

(3) $\int_{\Gamma \backslash \mathcal{H}}\left|f(z) \Im(z)^{\frac{m}{2}}\right|^{2} d v(z)<\infty$.

More generally, let $\chi: \Gamma \rightarrow \mathbb{C}^{\times}$be a character of finite order. The space $S_{m}(\Gamma, \chi)$ is defined as the space of all $f \in S_{m}(\operatorname{ker} \chi)$ that satisfy

$$
\left.f\right|_{m} \gamma=\chi(\gamma) f, \quad \gamma \in \Gamma \text {. }
$$

Clearly, $S_{m}(\Gamma)=S_{m}(\Gamma, 1)$. The space $S_{m}(\Gamma, \chi)$ is a finite-dimensional Hilbert space under the Petersson inner product

$$
\left\langle f_{1}, f_{2}\right\rangle_{\Gamma}:=\varepsilon_{\Gamma}^{-1} \int_{\Gamma \backslash \mathcal{H}} f_{1}(z) \overline{f_{2}(z)} \Im(z)^{m} d v(z), \quad f_{1}, f_{2} \in S_{m}(\Gamma, \chi) .
$$


Let us record the following basic lemma.

Lemma 3.2. Let $\sigma \in \mathrm{SL}_{2}(\mathbb{R})^{\sim}$. Then $\left.f \mapsto f\right|_{m} \sigma$ defines a unitary isomorphism $S_{m}(\Gamma, \chi) \rightarrow S_{m}\left(\sigma^{-1} \Gamma \sigma, \chi^{\sigma}\right)$, where $\chi^{\sigma}\left(\gamma^{\prime}\right):=\chi\left(\sigma \gamma^{\prime} \sigma^{-1}\right)$ for all $\gamma^{\prime} \in \sigma^{-1} \Gamma \sigma$.

The main results of this paper concern elements of $S_{m}(\Gamma, \chi)$ constructed in the form of a Poincaré series

$$
P_{\Lambda \backslash \Gamma, \chi} f:=\left.\sum_{\gamma \in \Lambda \backslash \Gamma} \overline{\chi(\gamma)} f\right|_{m} \gamma,
$$

where $\Lambda$ is a subgroup of $\Gamma$, and $f: \mathcal{H} \rightarrow \mathbb{C}$ satisfies $\left.f\right|_{m} \lambda=\chi(\lambda) f$ for all $\lambda \in \Lambda$. We write $P_{\Gamma, \chi} f:=P_{\{1\} \backslash \Gamma, \chi} f$ and $P_{\Gamma} f:=P_{\Gamma, 1} f$.

One checks easily that if $f \in S_{m}(\operatorname{ker} \chi)$, then $P_{\operatorname{ker} \chi \backslash \Gamma, \chi} f \in S_{m}(\Gamma, \chi)$, and we have

$$
\left\langle f_{1}, P_{\text {ker } \chi \backslash \Gamma, \chi} f\right\rangle_{\Gamma}=\left\langle f_{1}, f\right\rangle_{\text {ker } \chi}, \quad f_{1} \in S_{m}(\Gamma, \chi) .
$$

\section{Some Representation-theoretic Results}

Throughout this section, let $m \in \frac{3}{2}+\mathbb{Z}_{\geq 0}$.

Let $r$ be the right regular representation of $\mathrm{SL}_{2}(\mathbb{R})^{\sim}$ on $L^{2}\left(\mathrm{SL}_{2}(\mathbb{R})^{\sim}\right)$. For a discrete subgroup $\Gamma$ of $\mathrm{SL}_{2}(\mathbb{R})^{\sim}$, let $r_{\Gamma}$ be the unitary representation of $\mathrm{SL}_{2}(\mathbb{R})^{\sim}$ by right translations in $L^{2}\left(\Gamma \backslash \mathrm{SL}_{2}(\mathbb{R})^{\sim}\right)$.

Lemma 4.1. (1) There exists a unique (up to unitary equivalence) irreducible unitary representation $\overline{\pi_{m}}$ of $\mathrm{SL}_{2}(\mathbb{R})^{\sim}$ that decomposes, as a representation of $K$, into the orthogonal sum $\bigoplus_{k \in \mathbb{Z}_{\geq 0}} \chi_{m+2 k}$.

(2) Let $v$ be a non-zero element of the $\chi_{m}$-isotypic component of $\overline{\pi_{m}}$. Then, $\overline{\pi_{m}}\left(n^{-}\right) v=0$, and for every $k \in \mathbb{Z}_{\geq 0} \overline{\pi_{m}}\left(n^{+}\right)^{k} v$ spans the $\chi_{m+2 k^{-}}$ isotypic component of $\overline{\pi_{m}}$.

Proof. The claim (1) is [12, Lemma 3.5.(1)], and (2) is clear from the proof of [12, Lemma 3.5].

The following lemma is central to our proof of The inner product formula.

Lemma 4.2. Let $\Gamma$ be a discrete subgroup of $\mathrm{SL}_{2}(\mathbb{R})^{\sim}$. Suppose that $\varphi \in$ $C^{\infty}\left(\Gamma \backslash \mathrm{SL}_{2}(\mathbb{R})^{\sim}\right) \cap L^{2}\left(\Gamma \backslash \mathrm{SL}_{2}(\mathbb{R})^{\sim}\right), \varphi \not \equiv$, has the following properties:

(1) $\varphi$ transforms on the right by $K$ as $\chi_{m}$.

(2) $\mathcal{C} \varphi=m\left(\frac{m}{2}-1\right) \varphi$.

Then, the minimal closed subrepresentation $H$ of $r_{\Gamma}$ containing $\varphi$ is unitarily equivalent to $\overline{\pi_{m}}$, and $\varphi$ spans its $\chi_{m}$-isotypic component.

Proof. The lemma [1, Lemma 77] remains valid when the right regular representation of $G$ on $L^{2}(G)$ is replaced by the representation of $G$ by right translations in $L^{2}(\Lambda \backslash G)$, where $\Lambda$ is a discrete subgroup of $G$. By this result, 
$H$ is an orthogonal sum of finitely many closed irreducible $\mathrm{SL}_{2}(\mathbb{R}) \sim$-invariant subspaces. Hence, its $(\mathfrak{g}, K)$-module of $K$-finite vectors, $H_{K}$, is a direct sum of finitely many irreducible $(\mathfrak{g}, K)$-modules, and it is generated by $\varphi$ (see [2, Theorem 0.4]). From this it follows by an elementary computation in $H_{K}$, using (1)-(2), that $H_{K}$ is in fact an irreducible $(\mathfrak{g}, K)$-module and that it is isomorphic, as a $K$-module, to $\bigoplus_{k \in \mathbb{Z}_{\geq 0}} \chi_{m+2 k}$. Thus, $H$ is unitarily equivalent to $\overline{\pi_{m}}$ by Lemma 4.1.(1). Since $\varphi \not \equiv 0$ belongs to its (one-dimensional) $\chi_{m^{-}}$ isotypic component by (1), the second claim is clear.

Next, we recall the classical lift of $f: \mathcal{H} \rightarrow \mathbb{C}$ to $F_{f}: \mathrm{SL}_{2}(\mathbb{R})^{\sim} \rightarrow \mathbb{C}$ defined by

$$
F_{f}(\sigma):=\left(\left.f\right|_{m} \sigma\right)(i), \quad \sigma \in \mathrm{SL}_{2}(\mathbb{R})^{\sim},
$$

i.e., in Iwasawa coordinates,

$$
F_{f}\left(n_{x} a_{y} \kappa_{t}\right)=f(x+i y) y^{\frac{m}{2}} e^{-i m t}, \quad x, t \in \mathbb{R}, y \in \mathbb{R}_{>0} .
$$

The following result is well-known, but we could not find a convenient reference, so we provide a short proof.

THEOREM 4.3. Let $\Gamma$ be a discrete subgroup of finite covolume in $\mathrm{SL}_{2}(\mathbb{R})^{\sim}$. Then, the lift $f \mapsto F_{f}$ defines a unitary isomorphism $S_{m}(\Gamma) \rightarrow$ $\mathcal{A}\left(\Gamma \backslash \mathrm{SL}_{2}(\mathbb{R})^{\sim}\right)_{m}$, where $\mathcal{A}\left(\Gamma \backslash \mathrm{SL}_{2}(\mathbb{R})^{\sim}\right)_{m}$ is the subspace of $L^{2}\left(\Gamma \backslash \mathrm{SL}_{2}(\mathbb{R})^{\sim}\right)$ consisting of all $\varphi \in L^{2}\left(\Gamma \backslash \mathrm{SL}_{2}(\mathbb{R})^{\sim}\right) \cap C^{\infty}\left(\Gamma \backslash \mathrm{SL}_{2}(\mathbb{R})^{\sim}\right)$ with the following properties:

(1) $\varphi$ transforms on the right by $K$ as $\chi_{m}$.

(2) $\mathcal{C} \varphi=m\left(\frac{m}{2}-1\right) \varphi$.

Every $\varphi \in \mathcal{A}\left(\Gamma \backslash \mathrm{SL}_{2}(\mathbb{R})^{\sim}\right)_{m}$ is bounded.

Proof. An elementary computation using (4.1), (4.2), (2.7), (2.8), and Lemma 3.1 shows that $f \mapsto F_{f}$ is a well-defined isometry $S_{m}(\Gamma) \rightarrow$ $\mathcal{A}\left(\Gamma \backslash \mathrm{SL}_{2}(\mathbb{R})^{\sim}\right)_{m}$. To prove its surjectivity, let $\varphi \in \mathcal{A}\left(\Gamma \backslash \mathrm{SL}_{2}(\mathbb{R})^{\sim}\right)_{m}, \varphi \not \equiv 0$, and define $f: \mathcal{H} \rightarrow \mathbb{C}, f(x+i y):=\varphi\left(n_{x} a_{y}\right) y^{-\frac{m}{2}}$. Obviously, $f \in C^{\infty}(\mathcal{H})$ and $F_{f}=\varphi$. Next, by Lemma $4.2 \varphi$ spans the $\chi_{m}$-isotypic component of a closed subrepresentation of $r_{\Gamma}$ that is unitarily equivalent to $\overline{\pi_{m}}$. Thus, $n^{-} \varphi=0$ by Lemma 4.1.(2), so $\left(\partial_{x}+i \partial_{y}\right) f=0$ by $(2.10)$, hence $f$ is holomorphic. Furthermore, the fact that $\varphi \in L^{2}\left(\Gamma \backslash \mathrm{SL}_{2}(\mathbb{R})^{\sim}\right)$ implies that $f$ satisfies (3.1) and, by $(2.7)$, that $\int_{\Gamma \backslash \mathcal{H}}\left|f(z) \Im(z)^{\frac{m}{2}}\right|^{2} d v(z)<\infty$, so $f \in S_{m}(\Gamma)$ by Lemma 3.1. The same lemma implies that $\sup _{z \in \mathcal{H}}\left|f(z) \Im(z)^{\frac{m}{2}}\right|<\infty$, so $\varphi$ is bounded by $(4.2)$.

Next, let $\Gamma$ be a discrete subgroup of finite covolume in $\mathrm{SL}_{2}(\mathbb{R})^{\sim}$, and let $\chi$ be a character of $\Gamma$ of finite order. For $\varphi: \mathrm{SL}_{2}(\mathbb{R})^{\sim} \rightarrow \mathbb{C}$, we define the Poincaré series

$$
\left(P_{\Gamma, \chi} \varphi\right)(\sigma):=\sum_{\gamma \in \Gamma} \overline{\chi(\gamma)} \varphi(\gamma \sigma), \quad \sigma \in \mathrm{SL}_{2}(\mathbb{R})^{\sim}
$$


We write $P_{\Gamma} \varphi:=P_{\Gamma, 1} \varphi$. The following lemma provides an elementary connection between these Poincaré series and Poincaré series on $\mathcal{H}$.

Lemma 4.4. Let $f: \mathcal{H} \rightarrow \mathbb{C}$. Then the series $P_{\Gamma, \chi} f$ converges absolutely (resp., absolutely and uniformly on compact sets) on $\mathcal{H}$ if and only if $P_{\Gamma, \chi} F_{f}$ converges in the same way on $\mathrm{SL}_{2}(\mathbb{R})^{\sim}$, and in that case

$$
F_{P_{\Gamma, \chi} f}=P_{\Gamma, \chi} F_{f} \text {. }
$$

Proof. The claim follows by comparing (3.4) and the equality

$$
\begin{aligned}
\left(P_{\Gamma, \chi} F_{f}\right)\left(n_{x} a_{y} \kappa_{t}\right) & \stackrel{(4.3)}{=} \sum_{(4.1)} \overline{\chi(\gamma)}\left(\left.\left.f\right|_{m} \gamma\right|_{m} n_{x} a_{y} \kappa_{t}\right)(i) \\
& \stackrel{(2.4)}{=} \sum_{\gamma \in \Gamma} \overline{\chi(\gamma)}\left(\left.f\right|_{m} \gamma\right)(x+i y) y^{\frac{m}{2}} e^{-i m t},
\end{aligned}
$$

which holds for all $x, y, t \in \mathbb{R}$ with $y \in \mathbb{R}_{>0}$.

\section{Proof of The InNer Product Formula}

In this section, we prove the main result of this paper-The inner product formula (Theorem 5.4). We start by recalling a few results of [12] in the following lemma.

Lemma 5.1. Let $m \in \frac{3}{2}+\mathbb{Z}_{\geq 0}$ and $k \in \mathbb{Z}_{\geq 0}$.

(1) We define $f_{k, m}: \mathcal{H} \rightarrow \mathbb{C}$,

$$
f_{k, m}(z):=(2 i)^{m} \frac{(z-i)^{k}}{(z+i)^{m+k}} .
$$

$F_{k, m}:=F_{f_{k, m}}$ is a (unique up to a multiplicative constant) matrix coefficient of $\overline{\pi_{m}}$ that transforms on the right by $K$ as $\chi_{m}$ and on the left as $\chi_{m+2 k}$.

(2) $\mathcal{C} F_{k, m}=m\left(\frac{m}{2}-1\right) F_{k, m}$.

(3) We have

$F_{k, m}\left(\kappa_{\theta_{1}} h_{t} \kappa_{\theta_{2}}\right)=\chi_{m+2 k}\left(\kappa_{\theta_{1}}\right) \frac{\tanh ^{k}(t)}{\cosh ^{m}(t)} \chi_{m}\left(\kappa_{\theta_{2}}\right), \quad \theta_{1}, \theta_{2} \in \mathbb{R}, \quad t \in \mathbb{R}_{\geq 0}$.

(4) If $m \in \frac{5}{2}+\mathbb{Z}_{\geq 0}$, then $F_{k, m} \in L^{1}\left(\mathrm{SL}_{2}(\mathbb{R})^{\sim}\right)$.

(5) Suppose $m \in \frac{5}{2}+\mathbb{Z}_{\geq 0}$. Let $\Gamma$ be a discrete subgroup of finite covolume in $\mathrm{SL}_{2}(\mathbb{R})^{\sim}$. Then, the series $\sum_{\gamma \in \Gamma}\left|F_{k, m}(\gamma \cdot)\right|$ converges uniformly on compact sets in $\mathrm{SL}_{2}(\mathbb{R})^{\sim}$, and $P_{\Gamma} F_{k, m} \in \mathcal{A}\left(\Gamma \backslash \mathrm{SL}_{2}(\mathbb{R})^{\sim}\right)_{m}$.

Proof. The claim (1) is [12, Proposition 4.7], (2) follows from [12, Lemma 4.4.(3)], (3) is [12, Lemma 4.9], (4) is [12, Lemma 4.10], and (5) is clear from the proof of [12, Lemma 6.2].

Next, we prepare a few technical results for the proof of The inner product formula. 
Lemma 5.2. Let $m \in \frac{3}{2}+\mathbb{Z}_{\geq 0}$ and $k \in \mathbb{Z}_{\geq 0}$. Then, we have the following:

(1) $\left\|F_{k, m}\right\|_{L^{2}\left(\mathrm{SL}_{2}(\mathbb{R}) \sim\right)}^{2}=\frac{4 \pi k !}{\prod_{r=0}^{k}(m-1+r)}$.

(2) Let $f \in \operatorname{Hol}(\mathcal{H})$. Then, for all $x, y, t \in \mathbb{R}$ with $y>0$,

$$
\begin{aligned}
& \left(\left(n^{+}\right)^{k} F_{f}\right)\left(n_{x} a_{y} \kappa_{t}\right) \\
& \quad=\chi_{m+2 k}\left(\kappa_{t}\right) y^{\frac{m}{2}} \sum_{l=0}^{k}\left(\begin{array}{l}
k \\
l
\end{array}\right)(2 i y)^{l}\left(\prod_{r=l+1}^{k}(m-1+r)\right) f^{(l)}(x+i y) .
\end{aligned}
$$

(3) $\left(\left(n^{+}\right)^{k} F_{k, m}\right)(1)=k !$.

Proof. (1) By Lemma 5.1.(3) and (2.6), we have

$$
\left\|F_{k, m}\right\|_{L^{2}\left(\mathrm{SL}_{2}(\mathbb{R}) \sim\right)}^{2}=\frac{1}{4 \pi} \int_{0}^{4 \pi} \int_{0}^{\infty} \int_{0}^{4 \pi} \frac{\tanh ^{2 k}(t)}{\cosh ^{2 m}(t)} \sinh (2 t) d \theta_{1} d t d \theta_{2},
$$

which, substituting $x=\tanh ^{2}(t)$ and using the identities

$$
\sinh (2 t)=2 \sinh (t) \cosh (t) \quad \text { and } \quad \frac{1}{\cosh ^{2}(t)}=1-\tanh ^{2}(t)
$$

equals

$$
4 \pi \int_{0}^{1} x^{k}(1-x)^{m-2} d x=\frac{4 \pi k !}{\prod_{r=0}^{k}(m-1+r)} .
$$

The last equality is obtained by $k$-fold partial integration.

(2) This is proved by induction on $k \in \mathbb{Z}_{\geq 0}$ using (2.9) and noting that in the case when $k=0$ the equality (5.1) is the same as (4.2).

(3) Since $F_{k, m}=F_{f_{k, m}},(3)$ is just (2) applied to $f=f_{k, m}$ with $x=t=0$ and $y=1$.

Let $\Gamma$ be a discrete subgroup of $\mathrm{SL}_{2}(\mathbb{R})^{\sim}$. For $F \in L^{1}\left(\mathrm{SL}_{2}(\mathbb{R})^{\sim}\right)$ and $\varphi \in L^{2}\left(\Gamma \backslash \mathrm{SL}_{2}(\mathbb{R})^{\sim}\right), r_{\Gamma}(F) \varphi \in L^{2}\left(\Gamma \backslash \mathrm{SL}_{2}(\mathbb{R})^{\sim}\right)$ is standardly defined by the following condition:

$\left\langle r_{\Gamma}(F) \varphi, \phi\right\rangle_{L^{2}\left(\Gamma \backslash \mathrm{SL}_{2}(\mathbb{R})^{\sim}\right)}=\int_{\mathrm{SL}_{2}(\mathbb{R}) \sim} F(y)\left\langle r_{\Gamma}(y) \varphi, \phi\right\rangle_{L^{2}\left(\Gamma \backslash \mathrm{SL}_{2}(\mathbb{R})^{\sim}\right)} d \mu_{\mathrm{SL}_{2}(\mathbb{R}) \sim(y)}$ for all $\phi \in L^{2}\left(\Gamma \backslash \mathrm{SL}_{2}(\mathbb{R})^{\sim}\right)$. It is well-known that

$$
\left(r_{\Gamma}(F) \varphi\right)(x)=\int_{\mathrm{SL}_{2}(\mathbb{R}) \sim} F(y) \varphi(x y) d \mu_{\mathrm{SL}_{2}(\mathbb{R}) \sim(y)}
$$

for almost all $x \in \mathrm{SL}_{2}(\mathbb{R})^{\sim}$. The following lemma is immediate.

Lemma 5.3. Let $\Gamma$ be a discrete subgroup of $\mathrm{SL}_{2}(\mathbb{R})^{\sim}$. Let $F \in$ $L^{1}\left(\mathrm{SL}_{2}(\mathbb{R})^{\sim}\right)$ and $\varphi \in L^{2}\left(\Gamma \backslash \mathrm{SL}_{2}(\mathbb{R})^{\sim}\right)$. If $\varphi$ is continuous and bounded, then the integral in (5.2) is finite for every $x \in \mathrm{SL}_{2}(\mathbb{R})^{\sim}$, and the right-hand side of $(5.2)$ is a continuous function of $x \in \mathrm{SL}_{2}(\mathbb{R})^{\sim}$. 
Now we are ready to prove the main result of this paper.

ThEOREM 5.4 (The inner product formula). Let $\Gamma$ be a discrete subgroup of finite covolume in $\mathrm{SL}_{2}(\mathbb{R})^{\sim}$. Let $m \in \frac{5}{2}+\mathbb{Z}_{\geq 0}, k \in \mathbb{Z}_{\geq 0}$, and $\varphi \in \mathcal{A}\left(\Gamma \backslash \mathrm{SL}_{2}(\mathbb{R})^{\sim}\right)_{m}$. Then,

$$
\left\langle\varphi, P_{\Gamma} F_{k, m}\right\rangle_{L^{2}\left(\Gamma \backslash \mathrm{SL}_{2}(\mathbb{R}) \sim\right)}=\frac{4 \pi}{\prod_{r=0}^{k}(m-1+r)}\left(\left(n^{+}\right)^{k} \varphi\right)(1) .
$$

Proof. The case when $\varphi \equiv 0$ is trivial, so suppose that $\varphi \not \equiv 0$. We have

$$
\left\langle\varphi, P_{\Gamma} F_{k, m}\right\rangle_{L^{2}\left(\Gamma \backslash \mathrm{SL}_{2}(\mathbb{R}) \sim\right)}=\int_{\Gamma \backslash \mathrm{SL}_{2}(\mathbb{R}) \sim} \varphi(\sigma) \overline{\sum_{\gamma \in \Gamma} F_{k, m}(\gamma \sigma)} d \mu_{\Gamma \backslash \mathrm{SL}_{2}(\mathbb{R}) \sim(\sigma)}
$$

$$
\begin{aligned}
& =\int_{\Gamma \backslash \mathrm{SL}_{2}(\mathbb{R}) \sim} \sum_{\gamma \in \Gamma} \varphi(\gamma \sigma) \overline{F_{k, m}(\gamma \sigma)} d \mu_{\Gamma \backslash \mathrm{SL}_{2}(\mathbb{R}) \sim(\sigma)} \\
& =\int_{\mathrm{SL}_{2}(\mathbb{R}) \sim} \varphi \overline{F_{k, m}} d \mu_{\mathrm{SL}_{2}(\mathbb{R}) \sim}
\end{aligned}
$$

Now, $F_{k, m} \in L^{1}\left(\mathrm{SL}_{2}(\mathbb{R})^{\sim}\right)$ by Lemma 5.1.(4), and $\varphi$ is continuous and bounded by Theorem 4.3. Thus, by Lemma 5.3, $r_{\Gamma}\left(\overline{F_{k, m}}\right) \varphi \in$ $L^{2}\left(\Gamma \backslash \mathrm{SL}_{2}(\mathbb{R})^{\sim}\right) \cap C\left(\Gamma \backslash \mathrm{SL}_{2}(\mathbb{R})^{\sim}\right)$ is given by

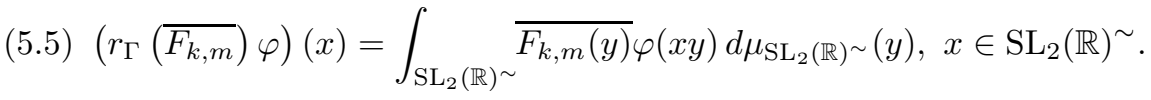

In particular,

$$
\left(r_{\Gamma}\left(\overline{F_{k, m}}\right) \varphi\right)(1)=\int_{\mathrm{SL}_{2}(\mathbb{R}) \sim} \varphi \overline{F_{k, m}} d \mu_{\mathrm{SL}_{2}(\mathbb{R}) \sim}
$$

so (5.4) implies that

$$
\left\langle\varphi, P_{\Gamma} F_{k, m}\right\rangle_{L^{2}\left(\Gamma \backslash \mathrm{SL}_{2}(\mathbb{R}) \sim\right)}=\left(r_{\Gamma}\left(\overline{F_{k, m}}\right) \varphi\right)(1) .
$$

To compute $\left(r_{\Gamma}\left(\overline{F_{k, m}}\right) \varphi\right)(1)$, we note that by Lemma $4.2 \varphi$ generates the $\chi_{m}$-isotypic component of a closed subrepresentation $H_{\varphi}$ of $r_{\Gamma}$ that is unitarily equivalent to $\overline{\pi_{m}}$. Clearly, $r_{\Gamma}\left(\overline{F_{k, m}}\right) \varphi \in H_{\varphi}$. In fact, $r_{\Gamma}\left(\overline{F_{k, m}}\right) \varphi$ belongs to the $\chi_{m+2 k}$-isotypic component of $H_{\varphi}$ : since $F_{k, m}$ transforms on the left by $K$ as $\chi_{m+2 k}$ by Lemma 5.1.(1), we have

$$
\begin{aligned}
&\left(r_{\Gamma}\left(\overline{F_{k, m}}\right) \varphi\right)(x \kappa) \stackrel{(5.5)}{=} \int_{\mathrm{SL}_{2}(\mathbb{R}) \sim} \overline{F_{k, m}(y)} \varphi(x \kappa y) d \mu_{\mathrm{SL}_{2}(\mathbb{R}) \sim(y)} \\
&=\int_{\mathrm{SL}_{2}(\mathbb{R}) \sim} \overline{F_{k, m}\left(\kappa^{-1} y\right)} \varphi(x y) d \mu_{\mathrm{SL}_{2}(\mathbb{R}) \sim}(y) \\
&=\chi_{m+2 k}(\kappa) \int_{\mathrm{SL}_{2}(\mathbb{R}) \sim} \overline{F_{k, m}(y)} \varphi(x y) d \mu_{\mathrm{SL}_{2}(\mathbb{R}) \sim(y)} \\
& \stackrel{(5.5)}{=} \chi_{m+2 k}(\kappa)\left(r_{\Gamma}\left(\overline{F_{k, m}} \varphi\right)(x)\right.
\end{aligned}
$$


for all $x \in \mathrm{SL}_{2}(\mathbb{R})^{\sim}$ and $\kappa \in K$. Hence, by Lemma 4.1.(2),

$$
r_{\Gamma}\left(\overline{F_{k, m}}\right) \varphi=\lambda\left(n^{+}\right)^{k} \varphi \quad \text { for some } \lambda \in \mathbb{C} .
$$

To calculate $\lambda$, we apply Lemma 4.2 , with $\Gamma=\{1\}$, to $F_{k, m}$. (The function $F_{k, m}$ satisfies all conditions of Lemma 4.2 by Lemmas 5.1.(1)-(2) and 5.2.(1).) We obtain that $F_{k, m}$ spans the $\chi_{m}$-isotypic component of a closed subrepresentation $H_{F_{k, m}}$ of $r$ that is unitarily equivalent to $\overline{\pi_{m}}$. Let $\Phi: H_{\varphi} \rightarrow H_{F_{k, m}}$ be a unitary equivalence. Since $\varphi$ and $F_{k, m}$ span the $\chi_{m^{-}}$ isotypic components of, respectively, $H_{\varphi}$ and $H_{F_{k, m}}$, we have

$$
\Phi \varphi=s F_{k, m} \quad \text { for some } s \in \mathbb{C}^{\times} .
$$

By applying $\Phi$ to both sides of (5.7), we obtain

$$
r\left(\overline{F_{k, m}}\right) \Phi \varphi=\lambda\left(n^{+}\right)^{k} \Phi \varphi,
$$

which by equality (5.8) implies the following equality of continuous functions $\mathrm{SL}_{2}(\mathbb{R})^{\sim} \rightarrow \mathbb{C}:$

$$
r\left(\overline{F_{k, m}}\right) F_{k, m}=\lambda\left(n^{+}\right)^{k} F_{k, m} .
$$

By evaluating these functions at $1 \in \mathrm{SL}_{2}(\mathbb{R})^{\sim}$ and using that

$$
\left(r\left(\overline{F_{k, m}}\right) F_{k, m}\right)(1)=\left\|F_{k, m}\right\|_{L^{2}\left(\mathrm{SL}_{2}(\mathbb{R}) \sim\right)}^{2}
$$

by (5.2) and Lemma 5.3, we obtain

$$
\lambda=\frac{\left\|F_{k, m}\right\|_{L^{2}\left(\mathrm{SL}_{2}(\mathbb{R}) \sim\right)}^{2}}{\left(\left(n^{+}\right)^{k} F_{k, m}\right)(1)}=\frac{4 \pi}{\prod_{r=0}^{k}(m-1+r)}
$$

by Lemma 5.2. (1) and (3). Thus,

$$
\begin{aligned}
\left\langle\varphi, P_{\Gamma} F_{k, m}\right\rangle_{L^{2}\left(\Gamma \backslash \mathrm{SL}_{2}(\mathbb{R}) \sim\right)} \stackrel{\stackrel{(5.6)}{=}\left(r_{\Gamma}\left(\overline{F_{k, m}}\right) \varphi\right)(1)}{ } & \stackrel{(5.7)}{=} \lambda\left(\left(n^{+}\right)^{k} \varphi\right)(1) \\
& \stackrel{(5.9)}{=} \frac{4 \pi}{\prod_{r=0}^{k}(m-1+r)}\left(\left(n^{+}\right)^{k} \varphi\right)(1) .
\end{aligned}
$$

6. The matrix COefficients SPAnning Set For the SPACE $S_{m}(\Gamma, \chi)$

Throughout this section, let $m \in \frac{5}{2}+\mathbb{Z}_{\geq 0}$.

THEOREM 6.1. Let $\Gamma$ be a discrete subgroup of finite covolume in $\mathrm{SL}_{2}(\mathbb{R})^{\sim}$, $\chi: \Gamma \rightarrow \mathbb{C}^{\times}$a character of finite order, and $k \in \mathbb{Z}_{\geq 0}$. Then:

(1) The series $P_{\Gamma, \chi} f_{k, m}$ converges absolutely and uniformly on compact sets in $\mathcal{H}$.

(2) $P_{\Gamma, \chi} f_{k, m} \in S_{m}(\Gamma, \chi)$. 
(3) For every $f \in S_{m}(\Gamma, \chi)$,

$$
\left\langle f, P_{\Gamma, \chi} f_{k, m}\right\rangle_{\Gamma}=\sum_{l=0}^{k}\left(\begin{array}{l}
k \\
l
\end{array}\right)(2 i)^{l} \frac{4 \pi}{\prod_{r=0}^{l}(m-1+r)} f^{(l)}(i) .
$$

(4) $\left\{P_{\Gamma, \chi} f_{n, m}: n \in \mathbb{Z}_{\geq 0}\right\}$ spans $S_{m}(\Gamma, \chi)$.

Proof. (1) By Lemma 4.4, it suffices to prove that the series $P_{\Gamma, \chi} F_{f_{k, m}}=$ $P_{\Gamma, \chi} F_{k, m}$ converges absolutely and uniformly on compact sets in $\mathrm{SL}_{2}(\mathbb{R}) \sim$, which is clear from Lemma 5.1.(5).

Next, we prove (2)-(4) in the case when $\chi=1$ :

(2) Since $F_{P_{\Gamma} f_{k, m}}=P_{\Gamma} F_{k, m}$ belongs to $\mathcal{A}\left(\Gamma \backslash \mathrm{SL}_{2}(\mathbb{R})^{\sim}\right)_{m}$ by Lemma 5.1.(5), it follows by Theorem 4.3 that $P_{\Gamma} f_{k, m}$ belongs to $S_{m}(\Gamma)$.

(3) Let $f \in S_{m}(\Gamma)$. We have, by Theorem 4.3,

$$
\begin{aligned}
\left\langle f, P_{\Gamma} f_{k, m}\right\rangle_{\Gamma} & =\left\langle F_{f}, F_{P_{\Gamma} f_{k, m}}\right\rangle_{L^{2}\left(\Gamma \backslash \mathrm{SL}_{2}(\mathbb{R}) \sim\right)} \\
& \stackrel{(4.4)}{=}\left\langle F_{f}, P_{\Gamma} F_{k, m}\right\rangle_{L^{2}\left(\Gamma \backslash \mathrm{SL}_{2}(\mathbb{R}) \sim\right)} \\
& \stackrel{(5.3)}{=} \frac{4 \pi}{\prod_{r=0}^{k}(m-1+r)}\left(\left(n^{+}\right)^{k} F_{f}\right)(1) \\
& \stackrel{(5.1)}{=} \sum_{l=0}^{k}\left(\begin{array}{l}
k \\
l
\end{array}\right)(2 i)^{l} \frac{4 \pi}{\prod_{r=0}^{l}(m-1+r)} f^{(l)}(i) .
\end{aligned}
$$

(4) It suffices to show that every $f \in S_{m}(\Gamma)$ satisfying $\left\langle f, P_{\Gamma} f_{n, m}\right\rangle_{\Gamma}=0$ for all $n \in \mathbb{Z}_{\geq 0}$ is identically zero. Indeed, from (3) it follows by induction on $n \in \mathbb{Z}_{\geq 0}$ that such an $f$ satisfies $f^{(n)}(i)=0$ for all $n \in \mathbb{Z}_{\geq 0}$, so $f$ is identically zero since $f \in \operatorname{Hol}(\mathcal{H})$.

Now, since

$$
P_{\Gamma, \chi} f_{k, m}=P_{\text {ker } \chi \backslash \Gamma, \chi} P_{\text {ker } \chi} f_{k, m},
$$

the claims $(2)-(4)$ in the case when $\chi \neq 1$ follow by (3.5) from the proven ones about $P_{\text {ker } \chi} f_{k, m}$.

Next, we give a result on the non-vanishing of cusp forms $P_{\Gamma, \chi} f_{k, m}$ in the case when $P(\Gamma) \subseteq \mathrm{SL}_{2}(\mathbb{Z})$. Let us denote by $\mathrm{M}(a, b)$ the median of the beta distribution with parameters $a, b \in \mathbb{R}_{>0}$, i.e., the unique $\left.\mathrm{M}(a, b) \in\right] 0,1[\operatorname{such}$ that

$$
\int_{0}^{\mathrm{M}(a, b)} x^{a-1}(1-x)^{b-1} d x=\int_{\mathrm{M}(a, b)}^{1} x^{a-1}(1-x)^{b-1} d x .
$$

Theorem 6.2. Let $N \in \mathbb{Z}_{>0}$ and $k \in \mathbb{Z}_{\geq 0}$. Let $\Gamma$ be a subgroup of finite index in $P^{-1}(\Gamma(N))$, and let $\chi: \Gamma \rightarrow \mathbb{C}^{\times}$be a character of finite order. Then, we have the following: 
(1) If

$$
\left.\chi\right|_{\Gamma \cap K} \neq\left.\chi_{m+2 k}\right|_{\Gamma \cap K}
$$

then $P_{\Gamma, \chi} f_{k, m} \equiv 0$.

(2) Suppose that

If

$$
\left.\chi\right|_{\Gamma \cap K}=\left.\chi_{m+2 k}\right|_{\Gamma \cap K} .
$$

$$
N>\frac{4 \mathrm{M}\left(\frac{k}{2}+1, \frac{m}{2}-1\right)^{\frac{1}{2}}}{1-\mathrm{M}\left(\frac{k}{2}+1, \frac{m}{2}-1\right)}=: N_{k, m},
$$

then $P_{\Gamma, \chi} f_{k, m}$ is not identically zero.

Proof. (1) Since the function $F_{k, m}$ transforms on the left by $K$ as $\chi_{m+2 k}$, we have

$$
\begin{aligned}
& \left(P_{\Gamma, \chi} F_{k, m}\right)(\sigma)=\sum_{\gamma \in \Gamma \cap K \backslash \Gamma} \sum_{\delta \in \Gamma \cap K} \overline{\chi(\delta \gamma)} F_{k, m}(\delta \gamma \sigma) \\
& (6.4)=\sum_{\gamma \in \Gamma \cap K \backslash \Gamma} \underbrace{\left(\sum_{\delta \in \Gamma \cap K} \overline{\chi(\delta)} \chi_{m+2 k}(\delta)\right)}_{=: S} \overline{\chi(\gamma)} F_{k, m}(\gamma \sigma), \quad \sigma \in \mathrm{SL}_{2}(\mathbb{R})^{\sim} .
\end{aligned}
$$

By (6.1), $S$ is the sum of values of a non-trivial character of a finite abelian group, hence equals 0 . Thus, the function $F_{P_{\Gamma, \chi} f_{k, m}}=P_{\Gamma, \chi} F_{k, m}$ vanishes identically, hence $P_{\Gamma, \chi} f_{k, m} \equiv 0$.

(2) It suffices to prove the non-vanishing of $F_{P_{\Gamma, \chi} f_{k, m}}=P_{\Gamma, \chi} F_{k, m}$. We do this by applying to $P_{\Gamma, \chi} F_{k, m}$ the non-vanishing criterion [8, Lemma 2-1] with $\Gamma_{1}=\{1\}$ and $\Gamma_{2}=\Gamma \cap K$ : The function $F_{k, m}$ satisfies the condition (1) of [8, Lemma 2-1] since it transforms on the left by $K$ as $\chi_{m+2 k}$ and (6.2) holds. A compact set $C$ satisfying the conditions (2)-(3) of [8, Lemma 2-1] can be found using (6.3) exactly as in the proof of [12, Proposition 6.7].

The Table 1 shows the minimal levels $N$ for which Theorem 6.2.(2) proves the non-vanishing of the series $P_{\Gamma, \chi} F_{k, m}$, for $m \in\left\{\frac{5}{2}, 3, \frac{7}{2}, \ldots, 8\right\}$ and $k \in\{0,1,2, \ldots, 18\}$. The table was generated using $\mathrm{R} 3.3 .2$ [10]. To further illustrate the strength of Theorem 6.2.(2), we can use some well-known properties of the median $\mathrm{M}(a, b)$ ([12, Lemma 6.12]) to obtain the following variant of [12, Corollary 6.18].

Corollary 6.3. Let $N \in \mathbb{Z}_{>0}$ and $k \in \mathbb{Z}_{\geq 0}$. Let $\Gamma$ be a subgroup of finite index in $P^{-1}(\Gamma(N))$. Let $\chi: \Gamma \rightarrow \mathbb{C}^{\times}$be a character of finite order such that (6.2) holds. Then, $P_{\Gamma, \chi} f_{k, m}$ is not identically zero if one of the following holds:

(1) $k=0$ and $N>4 \cdot 2^{\frac{1}{m-2}} \sqrt{4^{\frac{1}{m-2}}-1}$, 
TABLE 1. The minimal level $N=\left\lfloor N_{k, m}\right\rfloor+1$ for which Theorem 6.2.(2) proves the non-vanishing of the series $P_{\Gamma, \chi} F_{k, m}$, for $m \in\left\{\frac{5}{2}, 3, \frac{7}{2}, \ldots, 8\right\}$ and $k \in\{0,1,2, \ldots, 18\}$.

\begin{tabular}{|c|c|c|c|c|c|c|c|c|c|c|c|c|}
\hline $\mathrm{m}$ & $\frac{5}{2}$ & 3 & $\frac{7}{2}$ & 4 & $\frac{9}{2}$ & 5 & $\frac{11}{2}$ & 6 & $\frac{13}{2}$ & 7 & $\frac{15}{2}$ & 8 \\
\hline 0 & 62 & 14 & 8 & 6 & 5 & 4 & 4 & 4 & 3 & 3 & 3 & 3 \\
\hline 1 & 107 & 23 & 13 & 9 & 7 & 6 & 5 & 5 & 4 & 4 & 4 & 4 \\
\hline 2 & 151 & 32 & 17 & 12 & 9 & 8 & 7 & 6 & 6 & 5 & 5 & 5 \\
\hline 3 & 197 & 40 & 21 & 15 & 12 & 10 & 8 & 7 & 7 & 6 & 6 & 5 \\
\hline 4 & 242 & 49 & 26 & 18 & 14 & 11 & 10 & 9 & 8 & 7 & 7 & 6 \\
\hline 5 & 288 & 58 & 30 & 21 & 16 & 13 & 11 & 10 & 9 & 8 & 7 & 7 \\
\hline 6 & 334 & 67 & 35 & 24 & 18 & 15 & 13 & 11 & 10 & 9 & 8 & 8 \\
\hline 7 & 379 & 75 & 39 & 26 & 20 & 16 & 14 & 12 & 11 & 10 & 9 & 8 \\
\hline 8 & 425 & 84 & 43 & 29 & 22 & 18 & 15 & 13 & 12 & 11 & 10 & 9 \\
\hline 9 & 471 & 93 & 48 & 32 & 24 & 20 & 17 & 15 & 13 & 12 & 11 & 10 \\
\hline 10 & 516 & 102 & 52 & 35 & 27 & 22 & 18 & 16 & 14 & 13 & 12 & 11 \\
\hline 11 & 562 & 111 & 57 & 38 & 29 & 23 & 20 & 17 & 15 & 14 & 13 & 12 \\
\hline 12 & 608 & 119 & 61 & 41 & 31 & 25 & 21 & 18 & 16 & 15 & 13 & 12 \\
\hline 13 & 654 & 128 & 65 & 44 & 33 & 27 & 22 & 19 & 17 & 16 & 14 & 13 \\
\hline 14 & 699 & 137 & 70 & 47 & 35 & 28 & 24 & 21 & 18 & 16 & 15 & 14 \\
\hline 15 & 745 & 146 & 74 & 50 & 37 & 30 & 25 & 22 & 19 & 17 & 16 & 15 \\
\hline 16 & 791 & 154 & 79 & 52 & 39 & 32 & 27 & 23 & 20 & 18 & 17 & 15 \\
\hline 17 & 837 & 163 & 83 & 55 & 42 & 33 & 28 & 24 & 21 & 19 & 18 & 16 \\
\hline 18 & 882 & 172 & 87 & 58 & 44 & 35 & 29 & 25 & 22 & 20 & 18 & 17 \\
\hline
\end{tabular}

(2) $m=4$ and $N>\frac{4}{2^{\frac{1}{k+2}}-2^{-\frac{1}{k+2}}}$,

(3) $0<k \leq m-4$ and $N \geq 4 \sqrt{\frac{k+2}{m-2}\left(1+\frac{k+2}{m-2}\right)}$,

(4) $0<m-4 \leq k$ and $N \geq 4 \sqrt{\frac{k}{m-4}\left(1+\frac{k}{m-4}\right)}$.

7. The SPAnning Set For the SPaCe $S_{m}(\Gamma, \chi)$ Representing DERIVATIVES

Throughout this section, let $\Gamma$ be a discrete subgroup of finite covolume in $\mathrm{SL}_{2}(\mathbb{R})^{\sim}, \chi: \Gamma \rightarrow \mathbb{C}^{\times}$a character of finite order, and $m \in \frac{5}{2}+\mathbb{Z}_{\geq 0}$.

For every $k \in \mathbb{Z}_{\geq 0}$ and $\xi \in \mathcal{H}$, we define $\delta_{k, m, \xi}: \mathcal{H} \rightarrow \mathbb{C}$,

$$
\delta_{k, m, \xi}(z):=\frac{(2 i)^{m}}{4 \pi}\left(\prod_{r=0}^{k}(m-1+r)\right) \frac{1}{(z-\bar{\xi})^{m+k}} .
$$


Note that $\delta_{k, m, \xi}(z)=\left(\frac{d}{d \bar{\xi}}\right)^{k} \delta_{0, m, \xi}(z)$.

Proposition 7.1. Let $k \in \mathbb{Z}_{\geq 0}$ and $\xi \in \mathcal{H}$. Then, the Poincaré series

$\Delta_{\Gamma, k, m, \xi, \chi}(z):=\left(P_{\Gamma, \chi} \delta_{k, m, \xi}\right)(z)$

$$
=\frac{(2 i)^{m}}{4 \pi}\left(\prod_{r=0}^{k}(m-1+r)\right) \sum_{\gamma \in \Gamma} \frac{\overline{\chi(\gamma)}}{(\gamma . z-\bar{\xi})^{m+k}} \eta_{\gamma}(z)^{-2 m}
$$

converges absolutely and uniformly on compact sets in $\mathcal{H}$ and belongs to $S_{m}(\Gamma, \chi)$.

Proof. This can be proved by applying the obvious half-integral weight variant of [4, Theorems 2.6.6.(1) and 2.6.7]. We give an alternative proof. Note that

$$
f_{k, m}=\sum_{l=0}^{k}\left(\begin{array}{l}
k \\
l
\end{array}\right)(-2 i)^{l} \frac{4 \pi}{\prod_{r=0}^{l}(m-1+r)} \delta_{l, m, i}, \quad k \in \mathbb{Z}_{\geq 0},
$$

hence by the binomial inversion formula

$$
\delta_{k, m, i}=\frac{\prod_{r=0}^{k}(m-1+r)}{4 \pi(2 i)^{k}} \sum_{l=0}^{k}\left(\begin{array}{l}
k \\
l
\end{array}\right)(-1)^{l} f_{l, m}, \quad k \in \mathbb{Z}_{\geq 0},
$$

so the claim in the case when $\xi=i$ follows from Theorem 6.1.(1) and (2). Now the claim for general $\xi=x+i y \in \mathcal{H}$ (with $x, y \in \mathbb{R}$ ) is clear, using Lemma 3.2, from the identity

$$
\Delta_{\Gamma, k, m, \xi, \chi}=\left.y^{-\frac{m}{2}-k} \Delta_{\left(n_{x} a_{y}\right)^{-1} \Gamma n_{x} a_{y}, k, m, i, \chi^{n_{x} a_{y}}}\right|_{m}\left(n_{x} a_{y}\right)^{-1},
$$

which is easily checked by definitions.

The following technical lemmas will be used in our analytic proof of the fact that cusp forms $\Delta_{\Gamma, k, m, \xi, \chi}$ represent $k$ th derivative functionals $S_{m}(\Gamma, \chi) \rightarrow \mathbb{C}$ (Theorem 7.4).

Lemma 7.2. Let $(X, d x)$ be a measure space. Let $D$ be a domain in $\mathbb{C}$. Suppose that $f: D \times X \rightarrow \mathbb{C}$ is a measurable function with the following properties.

(1) For every $x \in X, f(\cdot, x)$ is holomorphic on $D$.

(2) For every circle $C \subseteq D, \int_{C \times X}|f(z, x)| d(z, x)<\infty$.

Then, $F: D \rightarrow \mathbb{C}$,

$$
F(z):=\int_{X} f(z, x) d x
$$

is well-defined and holomorphic on D, and we have

$$
F^{(k)}(z)=\left.\int_{X}\left(\frac{d}{d \zeta}\right)^{k} f(\zeta, x)\right|_{\zeta=z} d x, \quad z \in D, k \in \mathbb{Z}_{>0} .
$$


Proof. Without (7.5), this is [4, Lemma 6.1.5]. To prove (7.5), let $z \in D$ and fix $\delta \in \mathbb{R}_{>0}$ such that $\{\zeta \in \mathbb{C}:|\zeta-z| \leq \delta\} \subseteq D$. Let $k \in \mathbb{Z}_{>0}$. We have

$$
\begin{aligned}
F^{(k)}(z) & =\frac{k !}{2 \pi i} \int_{|\zeta-z|=\delta} \frac{F(\zeta)}{(\zeta-z)^{k+1}} d \zeta \\
& \stackrel{\text { (‥4) }}{=} \int_{X}\left(\frac{k !}{2 \pi i} \int_{|\zeta-z|=\delta} \frac{f(\zeta, x)}{(\zeta-z)^{k+1}} d \zeta\right) d x \\
& =\left.\int_{X}\left(\frac{d}{d \zeta}\right)^{k} f(\zeta, x)\right|_{\zeta=z} d x
\end{aligned}
$$

by applying the Cauchy integral formula for derivatives in the first and the last, and Fubini's theorem in the second equality.

Lemma 7.3. Let $f \in S_{m}(\Gamma, \chi)$. Then, the function $I_{f}: \mathcal{H} \rightarrow \mathbb{C}$,

$$
I_{f}(\xi):=\left\langle f, \Delta_{\Gamma, 0, m, \xi, \chi}\right\rangle_{\Gamma},
$$

is holomorphic, and $I_{f}^{(k)}(\xi)=\left\langle f, \Delta_{\Gamma, k, m, \xi, \chi}\right\rangle_{\Gamma}$ for all $\xi \in \mathcal{H}$ and $k \in \mathbb{Z}_{>0}$.

Proof. For every $k \in \mathbb{Z}_{\geq 0}$ and $\xi \in \mathcal{H}$,

$$
\begin{aligned}
& \left\langle f, \Delta_{\Gamma, k, m, \xi, \chi}\right\rangle_{\Gamma} \stackrel{(3.3)}{=} \varepsilon_{\Gamma}^{-1} \int_{\Gamma \backslash \mathcal{H}} f(z) \sum_{\gamma \in \Gamma} \chi(\gamma) \overline{\left(\left.\delta_{k, m, \xi}\right|_{m} \gamma\right)(z)} \Im(z)^{m} d v(z) \\
& \stackrel{(3.2)}{=} \varepsilon_{\Gamma}^{-1} \int_{\Gamma \backslash \mathcal{H}} \sum_{\gamma \in \Gamma}\left(\left.f\right|_{m} \gamma\right)(z) \overline{\left(\left.\delta_{k, m, \xi}\right|_{m} \gamma\right)(z)} \Im(z)^{m} d v(z) \\
& \stackrel{(2.2)}{=} \varepsilon_{\Gamma}^{-1} \int_{\Gamma \backslash \mathcal{H}} \sum_{\gamma \in \Gamma} f(\gamma . z) \overline{\delta_{k, m, \xi}(\gamma \cdot z)} \Im(\gamma \cdot z)^{m} d v(z) \\
& =\int_{\mathcal{H}} f(z) \overline{\delta_{k, m, \xi}(z)} \Im(z)^{m} d v(z) \\
& \stackrel{(7.1)}{=} \frac{(-2 i)^{m}}{4 \pi}\left(\prod_{r=0}^{k}(m-1+r)\right) \int_{\mathcal{H}} \frac{f(z)}{(\bar{z}-\xi)^{m+k}} \Im(z)^{m} d v(z) .
\end{aligned}
$$

The claim of the lemma follows from (7.6) by Lemma 7.2. The condition (2) of Lemma 7.2 is satisfied since

$$
\begin{aligned}
\int_{\mathcal{H}} \frac{|f(z)|}{|\bar{z}-\xi|^{m+k}} \Im(z)^{m} d v(z) \\
\quad \leq\left(\sup _{z \in \mathcal{H}}\left|f(z) \Im(z)^{\frac{m}{2}}\right|\right) \int_{\mathcal{H}} \frac{\Im(z)^{\frac{m}{2}}}{|z-\bar{\xi}|^{m+k}} d v(z) \\
\quad=\left(\sup _{z \in \mathcal{H}}\left|f(z) \Im(z)^{\frac{m}{2}}\right|\right) \int_{\mathcal{H}} \frac{\Im(z)^{\frac{m}{2}}}{|z+i|^{m+k}} d v(z) \frac{1}{\Im(\xi)^{\frac{m}{2}+k}}
\end{aligned}
$$


(applying the substitution $z \mapsto n_{\Re(\xi)} a_{\Im(\xi)} . z$ for the last equality), and the right-hand side is obviously bounded for $\xi$ in any circle $C \subseteq \mathcal{H}$.

THEOREM 7.4. We have

$$
\left\langle f, \Delta_{\Gamma, k, m, \xi, \chi}\right\rangle_{\Gamma}=f^{(k)}(\xi), \quad f \in S_{m}(\Gamma, \chi), k \in \mathbb{Z}_{\geq 0}, \quad \xi \in \mathcal{H} .
$$

For every $\xi \in \mathcal{H},\left\{\Delta_{\Gamma, k, m, \xi, \chi}: k \in \mathbb{Z}_{\geq 0}\right\}$ spans $S_{m}(\Gamma, \chi)$.

Proof. Using (7.2), Theorem 6.1.(3) can be written in the following way: for all $f \in S_{m}(\Gamma, \chi)$ and $k \in \mathbb{Z}_{\geq 0}$,

$$
\begin{aligned}
\sum_{l=0}^{k}\left(\begin{array}{l}
k \\
l
\end{array}\right)(2 i)^{l} \frac{4 \pi}{\prod_{r=0}^{l}(m-1+r)} & \left\langle f, \Delta_{\Gamma, l, m, i, \chi}\right\rangle_{\Gamma} \\
& =\sum_{l=0}^{k}\left(\begin{array}{l}
k \\
l
\end{array}\right)(2 i)^{l} \frac{4 \pi}{\prod_{r=0}^{l}(m-1+r)} f^{(l)}(i) .
\end{aligned}
$$

This implies, by induction on $k \in \mathbb{Z}_{\geq 0}$, that for all $f \in S_{m}(\Gamma, \chi)$ and $k \in \mathbb{Z}_{\geq 0}$,

$$
\left\langle f, \Delta_{\Gamma, k, m, i, \chi}\right\rangle_{\Gamma}=f^{(k)}(i) .
$$

From here, one can obtain (7.8) for general $\xi \in \mathcal{H}$ in two ways. The first is algebraic (cf. the proof of [9, Lemma 3-8]): Let $f \in S_{m}(\Gamma, \chi)$. Let us write $x:=\Re(\xi)$ and $y:=\Im(\xi)$. By taking the $k$ th derivative at $z=i$ of the both sides of the equality $f(x+y z)=y^{-\frac{m}{2}}\left(\left.f\right|_{m} n_{x} a_{y}\right)(z)$, we obtain, using Lemma 3.2 ,

$$
\begin{aligned}
f^{(k)}(\xi) & =y^{-\frac{m}{2}-k}\left(\left.f\right|_{m} n_{x} a_{y}\right)^{(k)}(i) \\
& \stackrel{(7.9)}{=} y^{-\frac{m}{2}-k}\left\langle\left. f\right|_{m} n_{x} a_{y}, \Delta_{\left(n_{x} a_{y}\right)^{-1} \Gamma n_{x} a_{y}, k, m, i, \chi^{n_{x} a_{y}}}\right\rangle_{\left(n_{x} a_{y}\right)^{-1} \Gamma n_{x} a_{y}} \\
& =\left\langle f, y^{-\frac{m}{2}-k} \Delta_{\left(n_{x} a_{y}\right)^{-1} \Gamma n_{x} a_{y}, k, m, i,\left.\chi^{n_{x} a_{y}}\right|_{m}}\left(n_{x} a_{y}\right)^{-1}\right\rangle_{\Gamma} \\
& \stackrel{(7.3)}{=}\left\langle f, \Delta_{\Gamma, k, m, \xi, \chi}\right\rangle_{\Gamma} .
\end{aligned}
$$

A second way to obtain (7.8) from (7.9) is analytic: Let $f \in S_{m}(\Gamma, \chi)$. By Lemma 7.3, (7.9) shows that

$$
I_{f}^{(k)}(i)=f^{(k)}(i), \quad k \in \mathbb{Z}_{\geq 0},
$$

i.e., $f$ and $I_{f}$ have the same Taylor expansion at $i$. Since both are holomorphic on $\mathcal{H}$, it follows by the uniqueness of analytic continuation that

$$
I_{f}^{(k)}(\xi)=f^{(k)}(\xi), \quad \xi \in \mathcal{H}, k \in \mathbb{Z}_{\geq 0},
$$

and this is (7.8) by Lemma 7.3.

The second claim of the theorem follows from (7.8) as in the proof of Theorem 6.1.(4).

The equalities (7.8) and (7.6) prove the following integral formula: 
Corollary 7.5. Let $f \in S_{m}(\Gamma, \chi)$. Then, for all $k \in \mathbb{Z}_{\geq 0}$ and $\xi \in \mathcal{H}$,

$$
f^{(k)}(\xi)=\frac{(-2 i)^{m}}{4 \pi}\left(\prod_{r=0}^{k}(m-1+r)\right) \int_{\mathcal{H}} \frac{f(z)}{(\bar{z}-\xi)^{m+k}} \Im(z)^{m} d v(z) .
$$

More generally, Corollary 7.5 holds for every $f \in \operatorname{Hol}(\mathcal{H})$ such that $\sup _{z \in \mathcal{H}}\left|f(z) \Im(z)^{\frac{m}{2}}\right|<\infty$. This follows from the half-integral weight version of [4, Theorem 6.2.2]. As a simple application of Corollary 7.5, we prove the following.

Corollary 7.6. Let $f \in S_{m}(\Gamma, \chi)$. Then, for every $k \in \mathbb{Z}_{\geq 0}$,

$$
\sup _{\xi \in \mathcal{H}}\left|f^{(k)}(\xi) \Im(\xi)^{\frac{m}{2}+k}\right|<\infty .
$$

PROOF. By Corollary 7.5 and (7.7),

$$
\begin{aligned}
\sup _{\xi \in \mathcal{H}} \mid & f^{(k)}(\xi) \Im(\xi)^{\frac{m}{2}+k} \mid \\
& \leq \frac{2^{m}}{4 \pi}\left(\prod_{r=0}^{k}(m-1+r)\right)\left(\sup _{z \in \mathcal{H}}\left|f(z) \Im(z)^{\frac{m}{2}}\right|\right) \int_{\mathcal{H}} \frac{\Im(z)^{\frac{m}{2}}}{|z+i|^{m+k}} d v(z),
\end{aligned}
$$

and the right-hand side is finite by Lemma 3.1 .

Now we can easily prove the following result (cf. [7, (1-5)]).

Proposition 7.7. Let $k \in \mathbb{Z}_{\geq 0}$. Then,

$$
\sup _{z, \xi \in \mathcal{H}} \Im(\xi)^{\frac{m}{2}+k} \Im(z)^{\frac{m}{2}}\left|\Delta_{\Gamma, k, m, \xi, \chi}(z)\right|<\infty .
$$

Proof. Let us fix an orthonormal basis $\left\{f_{1}, \ldots, f_{d}\right\}$ of $S_{m}(\Gamma, \chi)$. We have

$$
\Delta_{\Gamma, k, m, \xi, \chi}(z)=\sum_{l=1}^{d}\left\langle\Delta_{\Gamma, k, m, \xi, \chi}, f_{l}\right\rangle_{\Gamma} f_{l}(z) \stackrel{(7.8)}{=} \sum_{l=1}^{d} \overline{f_{l}^{(k)}(\xi)} f_{l}(z),
$$

hence

$$
\begin{aligned}
\sup _{z, \xi \in \mathcal{H}} \Im(\xi)^{\frac{m}{2}+k} \Im(z)^{\frac{m}{2}}\left|\Delta_{\Gamma, k, m, \xi, \chi}(z)\right| & \\
& \leq \sum_{l=1}^{d}\left(\sup _{\xi \in \mathcal{H}}\left|f_{l}^{(k)}(\xi) \Im(\xi)^{\frac{m}{2}+k}\right|\right)\left(\sup _{z \in \mathcal{H}}\left|f_{l}(z) \Im(z)^{\frac{m}{2}}\right|\right),
\end{aligned}
$$

and the right-hand side is finite by Corollary 7.6. 


\section{TWO EXPANSIONS OF CUSP FORMS REPRESENTING DERIVATIVES}

Throughout this section, let $\Gamma$ be a discrete subgroup of finite covolume in $\mathrm{SL}_{2}(\mathbb{R})^{\sim}, \chi: \Gamma \rightarrow \mathbb{C}^{\times}$a character of finite order, and $m \in \frac{5}{2}+\mathbb{Z}_{\geq 0}$. Moreover, suppose that $\infty$ is a cusp of $P(\Gamma)$ and that

$$
\eta_{\gamma}(z)^{-2 m}=\chi(\gamma), \quad \gamma \in \Gamma_{\infty}, z \in \mathcal{H}
$$

Let $h \in \mathbb{R}_{>0}$ such that

$$
Z\left(\mathrm{SL}_{2}(\mathbb{R})^{\sim}\right) \Gamma_{\infty}=Z\left(\mathrm{SL}_{2}(\mathbb{R})^{\sim}\right)\left\langle n_{h}\right\rangle .
$$

By the half-integral weight version of [4, Theorem 2.6.9], for every $n \in \mathbb{Z}_{>0}$ the classical Poincaré series

$$
\psi_{\Gamma, n, m, \chi}:=P_{\Gamma_{\infty} \backslash \Gamma, \chi} e^{2 \pi i n \dot{h}}
$$

converges absolutely and uniformly on compact sets in $\mathcal{H}$, and $\psi_{\Gamma, n, m, \chi} \in$ $S_{m}(\Gamma, \chi)$. Moreover, by the half-integral weight version of [4, Theorem 2.6.10], every $f \in S_{m}(\Gamma, \chi)$ has the following Fourier expansion:

$$
f(z)=\frac{\varepsilon_{\Gamma}(4 \pi)^{m-1}}{\Gamma(m-1) h^{m}} \sum_{n=1}^{\infty} n^{m-1}\left\langle f, \psi_{\Gamma, n, m, \chi}\right\rangle_{\Gamma} e^{2 \pi i n \frac{z}{h}}, \quad z \in \mathcal{H} .
$$

Here we use the standard notation for the gamma function: $\Gamma(x):=$ $\int_{0}^{\infty} t^{x-1} e^{-t} d t, x \in \mathbb{R}_{>0}$.

Theorem 8.2 provides the Fourier expansion of cusp forms $\Delta_{\Gamma, k, m, \xi, \chi}$ and their expansion in a series of classical Poincaré series. It is a half-integral weight variant of [6, Theorem 3-5]. Lemma 8.1 resolves the convergence issues of its proof.

We define a norm $\|\cdot\|_{\Gamma, 1}$ on $S_{m}(\Gamma, \chi)$ by

$$
\|f\|_{\Gamma, 1}:=\int_{\Gamma \backslash \mathcal{H}}\left|f(z) \Im(z)^{\frac{m}{2}}\right| d v(z), \quad f \in S_{m}(\Gamma, \chi) .
$$

Lemma 8.1. Let $k \in \mathbb{Z}_{\geq 0}$ and $\xi \in \mathcal{H}$. Then, the series

$$
\sum_{n=1}^{\infty} n^{m+k-1} e^{-2 \pi i n \frac{\bar{\xi}}{h}} \psi_{\Gamma, n, m, \chi}
$$

converges:

(1) absolutely in the norm $\|\cdot\|_{\Gamma, 1}$,

(2) absolutely and uniformly on compact sets in $\mathcal{H}$,

(3) in the topology of $S_{m}(\Gamma, \chi)$.

Proof. The claim (1) implies the absolute convergence of (8.2) at every $z \in \mathcal{H}$ by [4, Corollary 2.6.2]. The claim (1) also implies the rest of the claims (2) and (3) since $S_{m}(\Gamma, \chi)$ is finite-dimensional. 
To prove (1), observe that

$$
\begin{aligned}
& \left\|\psi_{\Gamma, n, m, \chi}\right\|_{\Gamma, 1} \leq \int_{\Gamma \backslash \mathcal{H}} \sum_{\gamma \in \Gamma_{\infty} \backslash \Gamma}\left|\left(\left.e^{2 \pi i n \frac{\dot{h}}{m}}\right|_{m} \gamma\right)(z) \Im(z)^{\frac{m}{2}}\right| d v(z) \\
& \stackrel{(2.2)}{=} \int_{\Gamma \backslash \mathcal{H}} \sum_{\gamma \in \Gamma_{\infty} \backslash \Gamma}\left|e^{2 \pi i n \frac{\gamma \cdot z}{h}} \Im(\gamma \cdot z)^{\frac{m}{2}}\right| d v(z)=\int_{\Gamma_{\infty} \backslash \mathcal{H}}\left|e^{2 \pi i n \frac{z}{h}} \Im(z)^{\frac{m}{2}}\right| d v(z) \\
& =\int_{0}^{h} \int_{0}^{\infty} e^{-2 \pi n \frac{y}{h}} y^{\frac{m}{2}-2} d y d x=h\left(\frac{h}{2 \pi n}\right)^{\frac{m}{2}-1} \Gamma\left(\frac{m}{2}-1\right), \\
& \text { so } \\
& \sum_{n=1}^{\infty}\left\|n^{m+k-1} e^{-2 \pi i n \frac{\bar{\xi}}{h}} \psi_{\Gamma, n, m, \chi}\right\|_{\Gamma, 1} \leq \frac{h^{\frac{m}{2}}}{(2 \pi)^{\frac{m}{2}-1}} \Gamma\left(\frac{m}{2}-1\right) \sum_{n=1}^{\infty} n^{\frac{m}{2}+k} e^{-2 \pi n \frac{\Im(\xi)}{h}},
\end{aligned}
$$

and the right-hand side is finite by d'Alembert's ratio test.

TheOrem 8.2. Let $k \in \mathbb{Z}_{\geq 0}$ and $\xi \in \mathcal{H}$. Then:

(1) $\Delta_{\Gamma, k, m, \xi, \chi}$ has the following Fourier expansion:

$$
\Delta_{\Gamma, k, m, \xi, \chi}(z)=\frac{\varepsilon_{\Gamma}(4 \pi)^{m-1}}{\Gamma(m-1) h^{m}} \sum_{n=1}^{\infty} n^{m-1} \overline{\psi_{\Gamma, n, m, \chi}^{(k)}(\xi)} e^{2 \pi i n \frac{z}{h}}, \quad z \in \mathcal{H}
$$

(2) We have

$\Delta_{\Gamma, k, m, \xi, \chi}(z)=\frac{\varepsilon_{\Gamma}(4 \pi)^{m-1}(-2 \pi i)^{k}}{\Gamma(m-1) h^{m+k}} \sum_{n=1}^{\infty} n^{m+k-1} e^{-2 \pi i n \frac{\bar{\xi}}{h}} \psi_{\Gamma, n, m, \chi}(z), \quad z \in \mathcal{H}$.

The right-hand side converges in $S_{m}(\Gamma, \chi)$ and absolutely and uniformly on compact sets in $\mathcal{H}$.

Proof. This can be proved analogously to the proof of $[6$, Theorem 3 5], all convergence issues being settled by Lemma 8.1. We provide a shorter proof.

(1) The equality (8.3) follows from (8.1) since $\left\langle\Delta_{\Gamma, k, m, \xi, \chi}, \psi_{\Gamma, n, m, \chi}\right\rangle_{\Gamma} \stackrel{(7.8)}{=}$ $\overline{\psi_{\Gamma, n, m, \chi}^{(k)}(\xi)}$.

(2) We have

$$
\begin{aligned}
\Delta_{\Gamma, k, m, \xi, \chi}(z) & \stackrel{(7.8)}{=}\left\langle\Delta_{\Gamma, k, m, \xi, \chi}, \Delta_{\Gamma, 0, m, z, \chi}\right\rangle_{\Gamma} \stackrel{(7.8)}{=} \overline{\Delta_{\Gamma, 0, m, z, \chi}^{(k)}(\xi)} \\
& \stackrel{(8.3)}{=} \frac{\varepsilon_{\Gamma}(4 \pi)^{m-1}}{\Gamma(m-1) h^{m}} \sum_{n=1}^{\infty} n^{m-1} \psi_{\Gamma, n, m, \chi}(z) \overline{\left(\frac{2 \pi i n}{h}\right)^{k} e^{2 \pi i n \frac{\xi}{h}}} \\
& =\frac{\varepsilon_{\Gamma}(4 \pi)^{m-1}(-2 \pi i)^{k}}{\Gamma(m-1) h^{m+k}} \sum_{n=1}^{\infty} n^{m+k-1} e^{-2 \pi i n \frac{\bar{\xi}}{h}} \psi_{\Gamma, n, m, \chi}(z), z \in \mathcal{H} .
\end{aligned}
$$

The convergence claim follows from Lemma 8.1. 
Now we can easily prove some bounds on the derivatives of classical Poincaré series (cf. [7, Theorem 1-2]).

Corollary 8.3. Let $k \in \mathbb{Z}_{\geq 0}$. Then,

$$
\sup _{\substack{\xi \in \mathcal{H}, n \in \mathbb{Z}>0}} n^{\frac{m}{2}-1} \Im(\xi)^{\frac{m}{2}+k}\left|\psi_{\Gamma, n, m, \chi}^{(k)}(\xi)\right|<\infty .
$$

Proof. Let us fix an orthonormal basis $\left\{f_{1}, f_{2}, \ldots, f_{d}\right\}$ of $S_{m}(\Gamma, \chi)$, and for each $l \in\{1,2, \ldots, d\}$ let $f_{l}(z)=\sum_{n=1}^{\infty} a_{n}\left(f_{l}\right) e^{2 \pi i n \frac{z}{h}}$ be the Fourier expansion of $f_{l}$. We have

$$
\Delta_{\Gamma, k, m, \xi, \chi}(z)=\sum_{l=1}^{d}\left\langle\Delta_{\Gamma, k, m, \xi, \chi}, f_{l}\right\rangle_{\Gamma} f_{l}(z)=\sum_{n=1}^{\infty}\left(\sum_{l=1}^{d} \overline{f_{l}^{(k)}(\xi)} a_{n}\left(f_{l}\right)\right) e^{2 \pi i n \frac{z}{h}}
$$

for all $z, \xi \in \mathcal{H}$, hence by Theorem 8.2.(1)

$$
\frac{\varepsilon_{\Gamma}(4 \pi)^{m-1}}{\Gamma(m-1) h^{m}} n^{m-1} \overline{\psi_{\Gamma, n, m, \chi}^{(k)}(\xi)}=\sum_{l=1}^{d} \overline{f_{l}^{(k)}(\xi)} a_{n}\left(f_{l}\right), \quad n \in \mathbb{Z}_{>0}, \quad \xi \in \mathcal{H} .
$$

Thus,

$$
\begin{aligned}
& \sup _{\substack{\xi \in \mathcal{H}, n \in \mathbb{Z}>0}} n^{\frac{m}{2}-1} \Im(\xi)^{\frac{m}{2}+k}\left|\psi_{\Gamma, n, m, \chi}^{(k)}(\xi)\right| \\
& \quad \leq \frac{\Gamma(m-1) h^{m}}{\varepsilon_{\Gamma}(4 \pi)^{m-1}} \sum_{l=1}^{d}\left(\sup _{\xi \in \mathcal{H}}\left|f_{l}^{(k)}(\xi) \Im(\xi)^{\frac{m}{2}+k}\right|\right)\left(\sup _{n \in \mathbb{Z}_{>0}} \frac{\left|a_{n}\left(f_{l}\right)\right|}{n^{\frac{m}{2}}}\right)
\end{aligned}
$$

and the right-hand side is finite by Corollary 7.6 and by the half-integral weight version of [4, Corollary 2.1.6].

\section{Application to Cusp forms for $\Gamma_{0}(N)$}

We define the automorphic factor $J: \Gamma_{0}(4) \times \mathcal{H} \rightarrow \mathbb{C}$,

$$
J(\gamma, z):=\frac{\Theta(\gamma . z)}{\Theta(z)}
$$

where $\Theta \in \operatorname{Hol}(\mathcal{H})$ is given by $\Theta(z):=\sum_{n \in \mathbb{Z}} e^{2 \pi i n^{2} z}$. An explicit formula for $J$ is given by [3, III.(4.2)]. It easily implies that for every $N \in 4 \mathbb{Z}_{>0}$

$$
\boldsymbol{\Gamma}_{0}(N):=\left\{(\gamma, J(\gamma, \cdot)): \gamma \in \Gamma_{0}(N) \cap \Gamma_{1}(4)\right\}
$$

is a discrete subgroup of finite covolume in $\mathrm{SL}_{2}(\mathbb{R}) \sim$.

Let $m \in \frac{5}{2}+\mathbb{Z}_{\geq 0}$ and $N \in 4 \mathbb{Z}_{>0}$. Let $\chi$ be an even Dirichlet character modulo $N$. We identify $\chi$ with the character of $\Gamma_{0}(N)$ given by

$$
\left(\begin{array}{ll}
a & b \\
c & d
\end{array}\right) \mapsto \chi(d) \quad \text { for all } \quad\left(\begin{array}{ll}
a & b \\
c & d
\end{array}\right) \in \Gamma_{0}(N)
$$


and with the character of $\boldsymbol{\Gamma}_{0}(N)$ given by $(\gamma, J(\gamma, \cdot)) \mapsto \chi(\gamma)$ for all $\gamma \in$ $\Gamma_{0}(N) \cap \Gamma_{1}(4)$. Finally, we define

$$
S_{m}(N, \chi):=S_{m}\left(\boldsymbol{\Gamma}_{0}(N), \chi\right)
$$

This definition of $S_{m}(N, \chi)$ is equivalent to the one given in [11]. (In [11], $S_{m}(N, \chi)$ is defined regardless of the parity of $\chi$, but turns out to be trivial if $\chi$ is odd.) The Petersson inner product on $S_{m}(N, \chi)$ is

$$
\left\langle f_{1}, f_{2}\right\rangle_{\Gamma_{0}(N)}=\int_{\Gamma_{0}(N) \backslash \mathcal{H}} f_{1}(z) \overline{f_{2}(z)} \Im(z)^{m} d v(z), \quad f_{1}, f_{2} \in S_{m}(N, \chi),
$$

and we have, for all $k \in \mathbb{Z}_{\geq 0}$ and $\xi, z \in \mathcal{H}$,

$\Delta_{\boldsymbol{\Gamma}_{0}(N), k, m, \xi, \chi}(z)=\frac{(2 i)^{m}}{8 \pi}\left(\prod_{r=0}^{k}(m-1+r)\right) \sum_{\gamma \in \Gamma_{0}(N)} \frac{\overline{\chi(\gamma)}}{(\gamma \cdot z-\bar{\xi})^{m+k}} J(\gamma, z)^{-2 m}$.

The group $\boldsymbol{\Gamma}_{0}(N)$ and the character $\chi$ satisfy the assumptions of the first paragraph of Section 8 , hence we have the classical Poincaré series

$\psi_{\boldsymbol{\Gamma}_{0}(N), n, m, \chi}(z)=\sum_{\gamma \in \Gamma_{0}(N)_{\infty} \backslash \Gamma_{0}(N)} \overline{\chi(\gamma)} e^{2 \pi i n \gamma \cdot z} J(\gamma, z)^{-2 m}, \quad z \in \mathcal{H}, n \in \mathbb{Z}_{>0}$,

and the cusp forms $\Delta_{\boldsymbol{\Gamma}_{0}(N), k, m, \xi, \chi}$ have the expansion (8.4) in a series of classical Poincaré series. As a final application of our results, in Corollary 9.1 we express the action of Hecke operators of half-integral weight on $\Delta_{\boldsymbol{\Gamma}_{0}(N), k, m, \xi, \chi}$ in terms of (8.4) (cf. [6, Lemma 5-8]).

For every prime number $p$, the action of the Hecke operator $T_{p^{2}, m, \chi}$ : $S_{m}(N, \chi) \rightarrow S_{m}(N, \chi)$ is given by the formula

$$
\sum_{n=1}^{\infty} a(n) e^{2 \pi i n z} \mid T_{p^{2}, m, \chi}:=\sum_{n=1}^{\infty} b(n) e^{2 \pi i n z},
$$

where the Fourier coefficients $b(n)$ are given by

$$
b(n):=a\left(p^{2} n\right)+\left(\frac{-1}{p}\right)^{m-\frac{1}{2}} \chi(p)\left(\frac{n}{p}\right) p^{m-\frac{3}{2}} a(n)+\chi\left(p^{2}\right) p^{2 m-2} a\left(n / p^{2}\right)
$$

([11, Theorem 1.7]). Here we understand that $a\left(n / p^{2}\right)=0$ if $p^{2} \nmid n$, while $(\dot{\bar{p}})$ is the usual Legendre symbol if $p$ is odd and is identically zero if $p=2$. If $p \nmid N$, then

$$
\left\langle f \mid T_{p^{2}, m, \chi}, g\right\rangle_{\boldsymbol{\Gamma}_{0}(N)}=\chi\left(p^{2}\right)\left\langle f, g \mid T_{p^{2}, m, \chi}\right\rangle_{\boldsymbol{\Gamma}_{0}(N)}, \quad f, g \in S_{m}(N, \chi) .
$$

This enables us to prove the following corollary. 
Corollary 9.1. Let $N \in 4 \mathbb{Z}_{>0}, m \in \frac{5}{2}+\mathbb{Z}_{\geq 0}, k \in \mathbb{Z}_{\geq 0}$, and $\xi \in \mathcal{H}$. Let $\chi$ be an even Dirichlet character modulo $N$. Then, for every prime number $p$ such that $p \nmid N, T_{p^{2}, m, \chi}$ maps the cusp form

$$
\Delta_{\Gamma_{0}(N), k, m, \xi, \chi}(z)=\frac{(4 \pi)^{m-1}(-2 \pi i)^{k}}{\Gamma(m-1)} \sum_{n=1}^{\infty} n^{m+k-1} e^{-2 \pi i n \bar{\xi}} \psi_{\Gamma_{0}(N), n, m, \chi}(z)
$$

to

$$
\begin{aligned}
& \left(\Delta_{\Gamma_{0}(N), k, m, \xi, \chi} \mid T_{p^{2}, m, \chi}\right)(z) \\
& \quad=\frac{(4 \pi)^{m-1}(-2 \pi i)^{k}}{\Gamma(m-1)} \sum_{n=1}^{\infty} n^{m+k-1} E_{p, k, n, m, \chi}(\xi) \psi_{\Gamma_{0}(N), n, m, \chi}(z),
\end{aligned}
$$

where

$$
\begin{aligned}
E_{p, k, n, m, \chi}(\xi):= & \operatorname{char}_{p^{2} \mathbb{Z}}(n) \frac{\chi\left(p^{2}\right)}{p^{2 k}} e^{-2 \pi i \frac{n}{p^{2}} \bar{\xi}} \\
& +\left(\frac{-1}{p}\right)^{m-\frac{1}{2}} \chi(p)\left(\frac{n}{p}\right) p^{m-\frac{3}{2}} e^{-2 \pi i n \bar{\xi}}+p^{2 m+2 k-2} e^{-2 \pi i p^{2} n \bar{\xi}} .
\end{aligned}
$$

Here char $p^{2} \mathbb{Z}$ is the characteristic function of $p^{2} \mathbb{Z} \subseteq \mathbb{Z}$, and $(\dot{\bar{p}})$ is the usual Legendre symbol.

Proof. The equality (9.3) is a special case of (8.4). The proof of (9.4) is analogous to that of $[6$, Lemma 5-8]. For every $z \in \mathcal{H}$, we have

$$
\begin{aligned}
\left(\Delta_{\boldsymbol{\Gamma}_{0}(N), k, m, \xi, \chi}\right. & \left.\mid T_{p^{2}, m, \chi}\right)(z) \stackrel{(7.8)}{=}\left\langle\Delta_{\boldsymbol{\Gamma}_{0}(N), k, m, \xi, \chi} \mid T_{p^{2}, m, \chi}, \Delta_{\boldsymbol{\Gamma}_{0}(N), 0, m, z, \chi}\right\rangle_{\boldsymbol{\Gamma}_{0}(N)} \\
& \stackrel{(9.2)}{=} \chi\left(p^{2}\right)\left\langle\Delta_{\boldsymbol{\Gamma}_{0}(N), k, m, \xi, \chi}, \Delta_{\boldsymbol{\Gamma}_{0}(N), 0, m, z, \chi} \mid T_{p^{2}, m, \chi}\right\rangle_{\boldsymbol{\Gamma}_{0}(N)} \\
& \stackrel{(7.8)}{=} \chi\left(p^{2}\right) \stackrel{\left(\Delta_{\boldsymbol{\Gamma}_{0}(N), 0, m, z, \chi} \mid T_{p^{2}, m, \chi}\right)^{(k)}(\xi)}{ }
\end{aligned}
$$

By (8.3) and (9.1), the right-hand side equals

$$
\begin{aligned}
{\left[\frac{(4 \pi)^{m-1}}{\Gamma(m-1)} \sum_{n=1}^{\infty}(-2 \pi i n)^{k}(\right.} & \chi\left(p^{2}\right)\left(p^{2} n\right)^{m-1} \psi_{\boldsymbol{\Gamma}_{0}(N), p^{2} n, m, \chi}(z) \\
& +\left(\frac{-1}{p}\right)^{m-\frac{1}{2}} \chi(p)\left(\frac{n}{p}\right) p^{m-\frac{3}{2}} n^{m-1} \psi_{\boldsymbol{\Gamma}_{0}(N), n, m, \chi}(z) \\
& \left.\left.+p^{2 m-2}\left(n / p^{2}\right)^{m-1} \psi_{\boldsymbol{\Gamma}_{0}(N), n / p^{2}, m, \chi}(z)\right) e^{-2 \pi i n \bar{\xi}}\right]
\end{aligned}
$$

By rearranging this sum to be over the index $n$ in $\psi_{\boldsymbol{\Gamma}_{0}(N), n, m, \chi}(z)$, we obtain (9.4). The rearrangement is valid by Lemma 8.1. 


\section{ACKNOWLEDGEMENTS}

This paper grew out of my PhD thesis. I would like to thank my advisor, Goran Muić, for his support, encouragement, and many discussons. I am also grateful to Neven Grbac and Marcela Hanzer for their continued support and useful comments. Finally, I would like to thank the referees, whose detailed suggestions helped to improve the style of exposition.

\section{REFERENCES}

[1] Harish-Chandra, Discrete series for semisimple Lie groups II. Explicit determination of the characters, Acta Math. 116 (1966), 1-111.

[2] A. W. Knapp and D. A. Vogan, Jr., Cohomological induction and unitary representations, Princeton University Press, Princeton, 1995.

[3] N. Koblitz, Introduction to elliptic curves and modular forms, Springer, New York, 2nd ed., 1993.

[4] T. Miyake, Modular forms, Springer, Berlin, 2006.

[5] G. Muić, On the cuspidal modular forms for the Fuchsian groups of the first kind, J. Number Theory 130 (2010), 1488-1511.

[6] G. Muić, Modular curves and bases for the spaces of cuspidal modular forms, Ramanujan J. 27 (2012), 181-208.

[7] G. Muić, On the analytic continuation and non-vanishing of L-functions, Int. J. Number Theory 8 (2012), 1831-1854.

[8] G. Muić, On the non-vanishing of certain modular forms, Int. J. Number Theory 7 (2011), 351-370.

[9] G. Muić, On the inner product of certain automorphic forms and applications, J. Lie Theory 22 (2012), 1091-1107.

[10] R Core Team, R: A language and environment for statistical computing. R Foundation for statistical computing, Vienna, Austria, 2016. https://www.R-project.org/.

[11] G. Shimura, On modular forms of half integral weight, Ann. of Math. (2) 97 (1973), 440-481.

[12] S. Žunar, On the non-vanishing of Poincaré series on the metaplectic group, to appear in Manuscripta Math. (2018). https://doi.org/10.1007/s00229-018-1011-6.

\section{S. Žunar}

Department of Mathematics

Faculty of Science

University of Zagreb

Bijenička 30, 10000 Zagreb

Croatia

E-mail: szunar@math.hr

Received: 20.11.2017.

Revised: 4.6.2018. 